\title{
Positive solutions of Kirchhoff type elliptic equations involving a critical Sobolev exponent
}

\author{
Daisuke Naimen
}

\begin{abstract}
In this paper we investigate the following Kirchhoff type elliptic boundary value problem involving a critical nonlinearity:$$
\begin{cases}-\left(a+b \int_{\Omega}|\nabla u|^{2} d x\right) \Delta u=\mu g(x, u)+u^{5}, u>0 & \text { in } \Omega, \\ u=0 & \text { on } \partial \Omega,\end{cases}
$$

here $\Omega \subset \mathbb{R}^{3}$ is a bounded domain with smooth boundary $\partial \Omega, a, b \geq 0$ and $a+b>0$. Under several conditions on $g \in C(\bar{\Omega} \times \mathbb{R}, \mathbb{R})$ and $\mu \in \mathbb{R}$, we prove the existence and nonexistence of solutions of (K1). This is some extension of a part of Brezis-Nirenberg's result in 1983.
\end{abstract}

Mathematics Subject Classification (2000). Primary 35J60; Secondary 35J20, 35J25.

Keywords. Kirchhoff, Elliptic, Critical, Variational method.

\section{Introduction}

We consider the following Kirchhoff type elliptic equation with a Dirichlet boundary condition:

$$
\begin{cases}-\left(a+b \int_{\Omega}|\nabla u|^{2} d x\right) \Delta u=\mu g(x, u)+u^{5} & \text { in } \Omega, \\ u>0 & \text { in } \Omega, \\ u=0 & \text { on } \partial \Omega,\end{cases}
$$

where $\Omega \subset \mathbb{R}^{3}$ is a bounded domain with smooth boundary $\partial \Omega$. We assume that $a, b \geq 0$ and $a+b>0$. In addition we emphasize that $u^{5}$ in the right hand side of the equation is a critical term, since 6 is a critical exponent in the sense of the Sobolev embedding $H_{0}^{1}(\Omega) \hookrightarrow L^{p}(\Omega)$. In this paper we prove the existence of solutions of (K1).

(K1) has its origin in the theory of nonlinear vibration. For instance, we give the following equation which describes the free vibration of a stretched string (cf. [27]): 


$$
\rho \frac{\partial^{2} u}{\partial t^{2}}=\left(T_{0}+\frac{E a}{2 L} \int_{0}^{L}\left|\frac{\partial u}{\partial x}\right|^{2} d x\right) \frac{\partial^{2} u}{\partial x^{2}},
$$

where $\rho>0$ is the mass per unit length, $T_{0}$ is the base tension, $E$ is the Young modulus, $a$ is the area of cross section and $L$ is the initial length of the string. (K0) takes account the change of the tension on the string which is caused by the change of its length during the vibration. The nonlocal equation of this type was first proposed by Kirchhoff in 1876 [19]. After that, several physicists also consider such equations for their researches in the theory of nonlinear vibrations theoretically or experimentally $[10,11,26,27,31]$. Moreover mathematically, the solvability of several Kirchhoff type quasilinear hyperbolic equations has been extensively discussed. See earlier results $[7,30]$ and the work by Lions [23], and recent ones [5,13]. For more details in the physical and mathematical background of Kirchhoff type equations, see the survey [4]. Recently, the Kirchhoff type elliptic equations such like (K1) get so many attentions. Many authors prove the existence of solutions of their problems using variational or topological methods. The main goal of their works is to study the effect of the non-local coefficients: $\left(a+b \int_{\Omega}|\nabla u|^{2} d x\right)$ (or in general denoted by $\left.m\left(\int_{\Omega}|\nabla u|^{2} d x\right)\right)$ on the principal term of their equations, on the existence results. See earlier results $[1,25][2,3,14,15,20-22,28,33,35]$ and so on.

On the other hand, as is well-known, when a nonlinear elliptic boundary value problem has a critical term such as (K1), a crucial difficulty occurs in proving the existence of solutions of the problem. Such difficulty is caused by the lack of compactness of the Sobolev embedding $H_{0}^{1}(\Omega) \hookrightarrow L^{6}(\Omega)$. Because of this difficulty, over these three decades, (K1) with $a=1$ and $b=0$ has been extensively studied by many authors. One of the most important results is obtained by Brezis and Nirenberg [9]. Our main aim in this paper is to extend their results in Section 2.5 in [9] to the case $a, b \geq 0$ and $a+b>0$. Compare the results below with those in Section 2.5 in [9].

To show our main results, we introduce some conditions on the function $g: \bar{\Omega} \times \mathbb{R} \rightarrow \mathbb{R}:$

(g1) $g$ is continuous in $\bar{\Omega} \times \mathbb{R}, g(x, u) \geq 0$ if $u \geq 0$ and $g(x, u)=0$ if $u \leq 0$ for all $x \in \Omega$.

(g2) $g(x, u)=o(u)$ as $u \rightarrow 0^{+}$and $g(x, u)=o\left(u^{5}\right)$ as $u \rightarrow \infty$ uniformly for $x \in \Omega$.

(g3) There exists a constant $\theta>0$ such that $4<\theta<6$ and $g(x, u) u-$ $\theta G(x, u) \geq 0$ for all $x \in \Omega$ and $u \geq 0$, where $G(x, u):=\int_{0}^{u} g(x, t) d t$.

(g4) There exists a nonempty open set $\omega \subset \Omega$ such that

$$
\lim _{u \rightarrow \infty} \frac{g(x, u)}{u^{3}}=\infty
$$

uniformly for $x \in \omega$.

The following theorem gives our main argument in this paper.

Theorem 1.1. Let $a, b \geq 0$ and $a+b>0$. Then if $g$ satisfies (g1)-(g4), (K1) has a solution for all $\mu>0$. 
In view of the typical power nonlinearities, Theorem 1.1 allows us to ensure the next corollary.

Corollary 1.2. Let $a, b \geq 0$ and $a+b>0$. Then if $3<q<5$ and $g(x, u)=u^{q}$, (K1) has a solution for all $\mu>0$.

Here we introduce the following assumptions (g5) and (g6) on $g$ which are weaker than (g3) and (g4) respectively.

(g5) There exists a constant $\theta>0$ such that $2<\theta<6$ and $g(x, u) u-$ $\theta G(x, u) \geq 0$ for all $x \in \Omega$ and $u \geq 0$.

(g6) There exist a nonempty open set $w \subset \Omega$ and an interval $I \subset(0, \infty)$ such that $g(x, u)>0$ if $x \in w$ and $u \in I$.

Now we can give the following theorem and corollary. An almost same result for (K1) with a more general nonlocal coefficient has already been obtained in [14].

Theorem 1.3. Let $a>0$ and $b \geq 0$. Then if $g$ satisfies (g1), (g2), (g5) and (g6), there exists a constant $\mu_{*} \geq 0$ such that (K1) has a positive solution for all $\mu>\mu_{*}$.

Corollary 1.4. Let $a>0$ and $b \geq 0$. Then if $1<q \leq 3$ and $g(x, u)=u^{q}$, there exists a constant $\mu_{*} \geq 0$ such that (K1) has a positive solution for all $\mu>\mu_{*}$.

As additional results for Corollaries 1.2 and 1.4, we can get the following nonexistence results. The first one is a Pohozaev type result [29].

Theorem 1.5. Let $a, b \geq 0, a+b>0$ and further, assume that $\mu \leq 0$ and $\Omega$ is strictly star-shaped. Then if $1<q<6$ and $g(x, u)=u^{q}$, (K1) has no solution.

The next one concludes that the value $\mu_{*}$ in Corollary 1.4 must be strictly positive if $\Omega$ is strictly star-shaped.

Theorem 1.6. In addition to the assumption of Corollary 1.4, we assume $\Omega \subset \mathbb{R}^{3}$ is strictly star-shaped. Then there exists a constant $\mu_{0}>0$, which is determined by $a, q$ and $\Omega$, such that (K1) has no solution for all $\mu \leq \mu_{0}$.

We can obtain these nonexistence results by slightly extension of the argument in [8] or [9]. For reader's convenience, we show the proof in Appendix A.

Recently some results related to (K1) are obtained in $[2,14,15,33]$. For (K1) with $\Omega=\mathbb{R}^{N}$, see $[3,22,35]$. In $[15,33]$, they consider the case $0<q<1$ and $g(x, u)=|u|^{q-1} u$. In [15], they show the existence of infinitely many (possibly sign-changing) solutions of (K1) for sufficiently small $\mu>0$. They use both the minimax theorem which is based on the Clark Theorem [12] and the second concentration compactness lemma by Lions [24]. In [33], they prove the existence of a positive solution of (K1) for sufficiently small $\mu>0$ by applying the method of the Nehari manifold and also using the second concentration compactness lemma. The results in $[2,14]$ are closely related to Theorem 1.3. In particular, in [14], they consider the general dimensional case, i.e., $N \geq 3$ and $\Omega \subset \mathbb{R}^{N}$. Using an appropriate truncation method, they obtain an almost same result with Theorem 1.3 for more general nonlocal 
problem. In view of these results, Theorem 1.1 is a new result for Kirchhoff type elliptic problems involving a critical Sobolev exponent. Although Theorem 1.3 has already almost proved in [14], inspired by [6,20], we introduce another truncation method and show the proof of Theorem 1.3 and Corollary 1.4 in Sect. 3. We also note that although our method can be applied to the general dimensional case as in $[6,20]$, in view of our main purpose of this paper, we only treat 3 dimensional case.

This paper is organized as follows. In Sect. 2, we prove Theorem 1.1 and Corollary 1.2. In Sect. 3, we prove Theorem 1.3 and Corollary 1.4. In Sect. 4, we consider (K1) with $a=0, b=1,1<q \leq 3$ and $g(x, u)=u^{q}$ as additional results. In Appendix A, we show the nonexistence results i.e., Theorem 1.5 and Theorem 1.6. In this paper we denote $H_{0}^{1}(\Omega)$ as a function space which is defined by the closure of $C_{0}^{\infty}(\Omega)$ in $H^{1}(\Omega)$. Thanks to the Poincare inequality, we define the usual $H_{0}^{1}(\Omega)$ norm as $\|u\|_{H_{0}^{1}(\Omega)}:=\left(\int_{\Omega}|\nabla u|^{2} d x\right)^{\frac{1}{2}}$. For simplicity we write $\|u\|=\|u\|_{H_{0}^{1}(\Omega)}$. Furthermore, we denote $H^{-1}(\Omega)$ as the dual space of $H_{0}^{1}(\Omega)$. We define the norm in $H^{-1}(\Omega)$ as $\|f\|_{H^{-1}(\Omega)}:=\sup _{\|u\| \leq 1}|\langle f, u\rangle|$.

Before beginning the proof, we define the weak solutions of $(\overline{\mathrm{K}} 1)$. We call $u \in H_{0}^{1}(\Omega)$ a weak solution of (K1) if and only if $u$ satisfies

$$
\left(a+b\|u\|^{2}\right) \int_{\Omega} \nabla u \cdot \nabla h d x-\mu \int_{\Omega} g(x, u) h d x-\int_{\Omega} u_{+}^{5} h d x=0
$$

for all $h \in H_{0}^{1}(\Omega)$. If $u \in H_{0}^{1}(\Omega)$ is a nontrivial weak solution for (K1), then we can trivially modify the usual elliptic regularity theorems (see for example, Lemma B.3 in [32] and the regularity theorems in [16]) and ensure the smoothness of $u$ up to $C^{2}(\bar{\Omega})$ even if $b>0$. Furthermore if $u$ is nonnegative, by the strong maximum principle, we have $u>0$ in $\Omega$. Consequently we can conclude that $u$ is a classical solution of (K1).

In the following sections we denote $C>0$ as some constants. If there occurs no confusion, we use same character $C$ even if the values of constants are different. Furthermore we denote $B(x, r) \subset \mathbb{R}^{3}$ as an open ball which is concentrated at $x$ with radius $r$.

\section{Proof of Theorem 1.1}

In this section, we prove Theorem 1.1 and Corollary 1.2. Our argument is based on that in [9]. Let $a, b \geq 0, a+b>0$ and fix $\mu>0$. Assume $g$ satisfies (g1)-(g4). We define the energy functional associated to (K1) so that

$$
I(u):=\frac{a}{2}\|u\|^{2}+\frac{b}{4}\|u\|^{4}-\mu \int_{\Omega} G(x, u) d x-\frac{1}{6} \int_{\Omega} u_{+}^{6} d x \quad\left(u \in H_{0}^{1}(\Omega)\right),
$$

where $G(x, u):=\int_{0}^{u} g(x, t) d t$ and $u_{+}:=\max \{u, 0\}$. Thanks to (g1) and (g2), $I$ is well-defined and continuously Fréchet differentiable on $H_{0}^{1}(\Omega)$. Furthermore every critical point of $I$ is a weak solution of (K1). Hence we shall find a nontrivial critical point of $I$. In the following argument, we often use the next fact which says, for all $\delta>0$ there exists a constant $C_{\delta}>0$ such that

$$
g(x, u) \leq \delta|u|^{5}+C_{\delta}|u|((x, u) \in \Omega \times \mathbb{R}) .
$$


This is a consequence of our hypotheses (g1) and (g2). We begin with the next lemmas.

Lemma 2.1. Let $g$ satisfies (g1) and (g2). Then there exist constants $\alpha, \rho>0$ such that

$$
I(u) \geq \alpha
$$

for all $u \in H_{0}^{1}(\Omega)$ with $\|u\|=\rho$.

Proof. By (g1) and (g2), there exists a constant $C>0$ such that

$$
g(x, t) \leq \frac{a \lambda_{1}}{4 \mu}|t|+C|t|^{5}
$$

for all $x \in \Omega$ and $t \in \mathbb{R}$, where $\lambda_{1}>0$ is the first eigenvalue of the problem:

$$
\begin{cases}-\Delta \phi=\lambda \phi & \text { in } \Omega, \\ \phi=0 & \text { on } \partial \Omega .\end{cases}
$$

Here we recall the well-known inequality:

$$
\int_{\Omega} u^{2} d x \leq \frac{1}{\lambda_{1}}\|u\|^{2} \quad\left(u \in H_{0}^{1}(\Omega)\right) .
$$

Take $u \in H_{0}^{1}(\Omega)$ with $\|u\|=\rho$. Using (2.2), (2.3) and the Sobolev embedding we get

$$
\begin{aligned}
I(u) & \geq \frac{a}{2}\|u\|^{2}+\frac{b}{4}\|u\|^{4}-\frac{a \lambda_{1}}{4} \int_{\Omega} u^{2} d x-C \int_{\Omega} u^{6} d x-\frac{1}{6} \int_{\Omega} u_{+}^{6} d x \\
& \geq \frac{a}{4}\|u\|^{2}+\frac{b}{4}\|u\|^{4}-C\|u\|^{6} \\
& =\frac{a}{4} \rho^{2}+\frac{b}{4} \rho^{4}-C \rho^{6}
\end{aligned}
$$

for some constant $C>0$. Since $a, b \geq 0$ and $a+b>0$, taking $\rho>0$ sufficiently small, we conclude that there exists a constant $\alpha>0$ such that

$$
I(u) \geq \alpha
$$

for all $u \in H_{0}^{1}(\Omega)$ with $\|u\|=\rho$. This completes the proof.

Lemma 2.2. Suppose $g$ satisfies (g1) and (g2). Then for every nontrivial function $v \in H_{0}^{1}(\Omega)$ with $v \geq 0$, there exists a constant $t_{0}>0$ such that if $v_{0}:=t_{0} v$, $\left\|v_{0}\right\|>\rho$ and $I\left(v_{0}\right) \leq 0$.

Proof. Take any nontrivial function $v \in H_{0}^{1}(\Omega)$ with $v \geq 0$. Note that by (g1), $G(x, s) \geq 0$ for all $x \in \Omega$ and $s \geq 0$. Using this fact, we have

$$
I(t v) \leq \frac{a t^{2}}{2}\|v\|^{2}+\frac{b t^{4}}{4}\|v\|^{4}-\frac{t^{6}}{6} \int_{\Omega} v^{6} d x .
$$

Then obviously, $I(t v) \rightarrow-\infty$ as $t \rightarrow \infty$ and thus, there exists a constant $t_{0}>0$ such that $I\left(t_{0} v\right) \leq 0$ and $\left\|t_{0} v\right\|>\rho$. This concludes the proof. 
Now we define

$$
\Gamma:=\left\{\gamma \in C\left([0,1], H_{0}^{1}(\Omega)\right) \mid \gamma(0)=0, I(\gamma(1)) \leq 0, \gamma(1) \neq 0\right\}
$$

and

$$
c:=\inf _{\gamma \in \Gamma} \max _{u \in \gamma([0,1])} I(u) .
$$

Note that from Lemma $2.2, \Gamma \neq \emptyset$. In addition, from the argument in Lemma 2.1 , clearly 0 is a local minimum of $I$ and $c \geq \alpha>0$. Consequently we have the existence of $(\mathrm{PS})_{c}$ sequences for $I$ (see Theorem 2.8 in [34] for instance). We prove the following lemma which is important to ensure the local compactness of PS sequences for $I$.

Lemma 2.3. Let g satisfy (g1), (g2) and (g3) and assume that $\left\{u_{j}\right\}$ is a $(P S)_{c}$ sequence for I with

$$
c<\frac{a}{2} C_{K}+\frac{b}{4} C_{K}^{2}-\frac{1}{6 S^{3}} C_{K}^{3},
$$

where

$$
C_{K}=\frac{1}{2}\left(b S^{3}+\sqrt{\left(b S^{3}\right)^{2}+4 a S^{3}}\right) .
$$

Then there exists a function $u \in H_{0}^{1}(\Omega)$ such that $\left(u_{j}\right)_{+} \rightarrow u_{+}$in $L^{6}(\Omega)$ up to subsequences.

Proof. Set

$$
c<\frac{a}{2} C_{K}+\frac{b}{4} C_{K}^{2}-\frac{1}{6 S^{3}} C_{K}^{3},
$$

and let $\left\{u_{j}\right\} \subset H_{0}^{1}(\Omega)$ be a $(\mathrm{PS})_{c}$ sequence for $I$. We first claim $\left\{u_{j}\right\}$ is bounded in $H_{0}^{1}(\Omega)$. In fact, since $I\left(u_{j}\right) \rightarrow c$ and $I^{\prime}\left(u_{j}\right) \rightarrow 0$ in $H^{-1}(\Omega)$, by $(\mathrm{g} 1)$ and (g3), we have

$$
\begin{aligned}
c+1 \geq & I\left(u_{j}\right)-\frac{1}{\theta}\left\langle I^{\prime}\left(u_{j}\right), u_{j}\right\rangle+\frac{1}{\theta}\left\langle I^{\prime}\left(u_{j}\right), u_{j}\right\rangle \\
\geq & a\left(\frac{1}{2}-\frac{1}{\theta}\right)\left\|u_{j}\right\|^{2}+b\left(\frac{1}{4}-\frac{1}{\theta}\right)\left\|u_{j}\right\|^{4} \\
& +\mu \int_{\Omega}\left(\frac{1}{\theta} g\left(x, u_{j}\right) u_{j}-G\left(x, u_{j}\right)\right) d x+\left(\frac{1}{\theta}-\frac{1}{6}\right) \int_{\Omega}\left(u_{j}\right)_{+}^{6} d x-\left\|u_{j}\right\| \\
\geq & a\left(\frac{1}{2}-\frac{1}{\theta}\right)\left\|u_{j}\right\|^{2}+b\left(\frac{1}{4}-\frac{1}{\theta}\right)\left\|u_{j}\right\|^{4}-\left\|u_{j}\right\|
\end{aligned}
$$

for large $j \in \mathbb{N}$. Since $a, b \geq 0$ and $a+b>0$, we can ensure the claim. Hence by the weak compactness of $H_{0}^{1}(\Omega)$ and the compactness of the Sobolev embeddings, there exists a function $u \in H_{0}^{1}(\Omega)$ such that

$$
\begin{aligned}
& u_{j} \rightarrow u \text { weakly in } H_{0}^{1}(\Omega), \\
& u_{j} \rightarrow u \text { in } L^{p}(\Omega) \text { for all } 1 \leq p<6, \\
& u_{j} \rightarrow u \text { a.e. on } \Omega,
\end{aligned}
$$

up to subsequences but still denoted $\left\{u_{j}\right\}$. Moreover from second concentration compactness lemma by Lions [24], there exist an at most countable set $\mathcal{J}$, 
points $\left\{x_{k}\right\}_{k \in \mathcal{J}} \subset \bar{\Omega}$ and values $\left\{\eta_{k}\right\}_{k \in \mathcal{J}},\left\{\nu_{k}\right\}_{k \in \mathcal{J}} \subset \mathbb{R}^{+}$such that up to subsequences,

$$
\begin{aligned}
& \left|\nabla u_{j}\right|^{2} \rightarrow d \eta \geq|\nabla u|^{2}+\sum_{k \in \mathcal{J}} \eta_{k} \delta_{x_{k}}, \\
& \left(u_{j}\right)_{+}^{6} \rightarrow d \nu=u_{+}^{6}+\sum_{k \in \mathcal{J}} \nu_{k} \delta_{x_{k}},
\end{aligned}
$$

in the measure sense, here $\delta_{x}$ is the Dirac delta measure concentrated at $x \in \mathbb{R}^{3}$ with mass 1 . In addition we also have the inequality

$$
\eta_{k} \geq S \nu_{k}^{\frac{1}{3}}(k \in \mathcal{J})
$$

Now we claim that $\mathcal{J}=\emptyset$. To ensure this, we suppose on the contrary $\mathcal{J} \neq \emptyset$. Fix $k \in \mathcal{J}$. Define a smooth function $\phi$ such that $\phi=1$ on $B\left(x_{k}, \varepsilon\right), \phi=0$ on $B\left(x_{k}, 2 \varepsilon\right)^{c}$ and $0 \leq \phi \leq 1$ otherwise. In addition we can assume that $|\nabla \phi| \leq 2 / \varepsilon$. As $I^{\prime}\left(u_{j}\right) \rightarrow 0$ in $H^{-1}(\Omega)$, we have

$$
\begin{aligned}
0= & \lim _{j \rightarrow \infty}\left\langle I^{\prime}\left(u_{j}\right), u_{j} \phi\right\rangle \\
= & \lim _{j \rightarrow \infty}\left\{\left(a+b\left\|u_{j}\right\|^{2}\right) \int_{\Omega} \nabla u_{j} \cdot \nabla\left(u_{j} \phi\right) d x-\mu \int_{\Omega} g\left(x, u_{j}\right) u_{j} \phi d x\right\} \\
& -\lim _{j \rightarrow \infty} \int_{\Omega}\left(u_{j}\right)_{+}^{6} \phi d x \\
= & \lim _{j \rightarrow \infty}\left\{\left(a+b\left\|u_{j}\right\|^{2}\right) \int_{\Omega}\left|\nabla u_{j}\right|^{2} \phi d x-\int_{\Omega}\left(u_{j}\right)_{+}^{6} \phi d x\right\}+o(1),
\end{aligned}
$$

where $o(1) \rightarrow 0$ as $\varepsilon \rightarrow 0$. The last equality comes from the facts that

$$
\lim _{j \rightarrow \infty}\left(a+b\left\|u_{j}\right\|^{2}\right) \int_{\Omega}\left(\nabla u_{j} \cdot \nabla \phi\right) u_{j} d x=o(1) \text { as } \varepsilon \rightarrow 0,
$$

and

$$
\lim _{j \rightarrow \infty} \int_{\Omega} g\left(x, u_{j}\right) u_{j} \phi d x=o(1) \text { as } \varepsilon \rightarrow 0 .
$$

We first verify (2.6). Actually, noting the boundedness and $L^{2}(\Omega)$ convergence of $\left\{u_{j}\right\}$ and using the Schwartz and the Hölder inequality we have

$$
\begin{aligned}
& \lim _{j \rightarrow \infty}\left(a+b\left\|u_{j}\right\|^{2}\right) \int_{\Omega}\left(\nabla u_{j} \cdot \nabla \phi\right) u_{j} d x \\
& \leq C \lim _{j \rightarrow \infty}\left(\int_{\Omega}\left|\nabla u_{j}\right|^{2} d x\right)^{\frac{1}{2}}\left(\int_{\Omega}\left|u_{j}\right|^{2}|\nabla \phi|^{2}\right)^{\frac{1}{2}} \\
& \leq C\left(\int_{\Omega \cap B\left(x_{k}, 2 \varepsilon\right)} u^{6} d x\right)^{\frac{1}{6}}\left(\int_{\Omega \cap B\left(x_{k}, 2 \varepsilon\right)}|\nabla \phi|^{3} d x\right)^{\frac{1}{3}} \\
& \leq C\left(\int_{\Omega \cap B\left(x_{k}, 2 \varepsilon\right)} u^{6} d x\right)^{\frac{1}{6}} \rightarrow 0 \text { as } \varepsilon \rightarrow 0,
\end{aligned}
$$


where for the last inequality we use our assumption $|\nabla \phi| \leq 2 / \varepsilon$. This ensures (2.6). We next verify (2.7). By (g1) and (g2), we have the inequality (2.1). Using that, we get

$$
\begin{aligned}
& \lim _{j \rightarrow \infty} \int_{\Omega} g\left(x, u_{j}\right) u_{j} \phi d x \\
& \quad \leq \lim _{j \rightarrow \infty}\left(\delta \int_{\Omega \cap B\left(x_{k}, 2 \varepsilon\right)} u_{j}^{6} \phi d x+C_{\delta} \int_{\Omega \cap B\left(x_{k}, 2 \varepsilon\right)} u_{j}^{2} \phi d x\right) \\
& \quad \leq C \delta+C_{\delta} \int_{\Omega \cap B\left(x_{k}, 2 \varepsilon\right)} u^{2} \phi d x,
\end{aligned}
$$

for some constant $C>0$, where for the last inequality we use the Sobolev embedding, the boundedness and the $L^{2}(\Omega)$ convergence of $\left\{u_{j}\right\}$. Hence we have

$$
\limsup _{\varepsilon \rightarrow 0}\left\{\lim _{j \rightarrow \infty} \int_{\Omega} g\left(x, u_{j}\right) u_{j} \phi d x\right\} \leq C \delta
$$

for all $\delta>0$. This proves (2.7). From (2.5), we have

$$
0 \geq\left\{\left(a+b \int_{\bar{\Omega}} \phi d \eta\right) \int_{\bar{\Omega}} \phi d \eta-\int_{\bar{\Omega}} \phi d \nu\right\}+o(1) \quad \text { as } \varepsilon \rightarrow 0 .
$$

Taking $\varepsilon \rightarrow 0$, we have

$$
0 \geq\left(a+b \eta_{k}\right) \eta_{k}-\nu_{k}
$$

Considering (2.4) together with (2.8), we get

$$
0 \geq\left(a+b \eta_{k}\right) \eta_{k}-\left(\frac{\eta_{k}}{S}\right)^{3} .
$$

Solving this inequality with respect to $\eta_{k}$, we estimate

$$
\eta_{k} \geq \frac{1}{2}\left(b S^{3}+\sqrt{\left(b S^{3}\right)^{2}+4 a S^{3}}\right)=C_{K} .
$$

On the other hand, again using (2.4) together with (2.8), we have

$$
0 \geq\left(a+b S \nu_{k}^{\frac{1}{3}}\right) S \nu_{k}^{\frac{1}{3}}-\nu_{k}
$$

Solving this with respect to $\nu_{k}$, we obtain

$$
\nu_{k} \geq \frac{C_{K}^{3}}{S^{3}} .
$$

Since $I\left(u_{j}\right) \rightarrow c$ and $I^{\prime}\left(u_{j}\right) \rightarrow 0$ in $H^{-1}(\Omega)$, recalling (g1) and (g3), we get

$$
\begin{aligned}
c= & \lim _{j \rightarrow \infty}\left\{I\left(u_{j}\right)-\frac{1}{\theta}\left\langle I^{\prime}\left(u_{j}\right), u_{j}\right\rangle\right\} \\
\geq & \lim _{j \rightarrow \infty}\left\{a\left(\frac{1}{2}-\frac{1}{\theta}\right)\left\|u_{j}\right\|^{2}+b\left(\frac{1}{4}-\frac{1}{\theta}\right)\left\|u_{j}\right\|^{4}\right\} \\
& +\lim _{j \rightarrow \infty}\left\{\mu \int_{\Omega}\left(\frac{1}{\theta} g\left(x, u_{j}\right)-G\left(x, u_{j}\right)\right) d x+\left(\frac{1}{\theta}-\frac{1}{6}\right) \int_{\Omega}\left(u_{j}\right)_{+}^{6} d x\right\} .
\end{aligned}
$$


Using (2.9) and (2.10), we have

$$
\begin{aligned}
c & \geq a\left(\frac{1}{2}-\frac{1}{\theta}\right) \eta_{k}+b\left(\frac{1}{4}-\frac{1}{\theta}\right) \eta_{k}^{2}+\left(\frac{1}{\theta}-\frac{1}{6}\right) \nu_{k} \\
& \geq a\left(\frac{1}{2}-\frac{1}{\theta}\right) C_{K}+b\left(\frac{1}{4}-\frac{1}{\theta}\right) C_{K}^{2}+\frac{1}{S^{3}}\left(\frac{1}{\theta}-\frac{1}{6}\right) C_{K}^{3} \\
& =\frac{a}{2} C_{K}+\frac{b}{4} C_{K}^{2}-\frac{1}{6 S^{3}} C_{K}^{3}
\end{aligned}
$$

where the last equality comes from the fact that $a C_{K}+b C_{K}^{2}-C_{K}^{3} / S^{3}=0$. This is a contradiction. Hence $\mathcal{J}=\emptyset$. Consequently we have

$$
\int_{\Omega}\left(u_{j}\right)_{+}^{6} d x \rightarrow \int_{\Omega} u_{+}^{6} d x \quad \text { as } j \rightarrow \infty .
$$

This leads us to the conclusion.

Remark 2.4. We can easily check that $u$ is nonnegative. In fact, since $\left\{u_{j}\right\}$ is bounded, there exist a subsequence, still denoted $\left\{u_{j}\right\}$, and a constant $A \geq 0$ such that $\left\|u_{j}\right\|^{2} \rightarrow A$. If $A=0$ the conclusion follows. If $A>0$, considering the weak convergence, (g1), (g2), and $L^{p}(\Omega)$ convergence for all $1 \leq p<6$, we have

$$
(a+b A) \int_{\Omega} \nabla u \cdot \nabla h d x=\int_{\Omega} g(x, u) h d x-\int_{\Omega} u_{+}^{5} h d x \quad\left(h \in H_{0}^{1}(\Omega)\right) .
$$

Taking $h=u_{-}$, where $u_{-}:=-\min \{0, u\}$ and considering (g1), we have

$$
\int_{\Omega}\left|\nabla u_{-}\right|^{2} d x=0
$$

The conclusion follows.

We ensure the local PS condition for $I$.

Lemma 2.5. Let g satisfy (g1)-(g3) and assume

Then I satisfies the $(\mathrm{PS})_{c}$ condition.

$$
c<\frac{a}{2} C_{K}+\frac{b}{4} C_{K}^{2}-\frac{1}{6 S^{3}} C_{K}^{3} .
$$

Proof. Let $\left\{u_{j}\right\}$ be a (PS)c sequence for $I$. Then by (g1) and (g2), we have that $\left\{u_{j}\right\}$ is bounded as in the proof of Lemma 2.3. Furthermore, by Lemma 2.3 and Remark 2.4, there exists a nonnegative function $u \in H_{0}^{1}(\Omega)$ such that

$$
\begin{aligned}
& u_{j} \rightarrow u \text { weakly in } H_{0}^{1}(\Omega), \\
& u_{j} \rightarrow u \text { in } L^{p}(\Omega) \text { for all } 1 \leq p<6, \\
& \left(u_{j}\right)_{+} \rightarrow u \text { in } L^{6}(\Omega),
\end{aligned}
$$

up to subsequences, but still denoted $\left\{u_{j}\right\}$. Since $I^{\prime}\left(u_{j}\right) \rightarrow 0$ in $H^{-1}(\Omega)$ and $\left\{u_{j}\right\}$ is bounded, we have

$$
\left\langle I^{\prime}\left(u_{j}\right),\left(u_{j}-u\right)\right\rangle=o(1)
$$

where $o(1) \rightarrow 0$ as $j \rightarrow \infty$. Here we claim

$$
\int_{\Omega} g\left(x, u_{j}\right)\left(u_{j}-u\right) d x=o(1) \quad \text { as } j \rightarrow \infty
$$


and

$$
\int_{\Omega}\left(u_{j}\right)_{+}^{5}\left(u_{j}-u\right) d x=o(1) \quad \text { as } j \rightarrow \infty .
$$

We first verify (2.12). Using (2.1) and the Hölder and the Schwartz inequality we have

$$
\begin{aligned}
& \limsup _{j \rightarrow \infty}\left|\int_{\Omega} g\left(x, u_{j}\right)\left(u_{j}-u\right) d x\right| \\
& \quad \leq \limsup _{j \rightarrow \infty}\left(\delta \int_{\Omega}\left|u_{j}\right|^{5}\left|u_{j}-u\right| d x+C_{\delta} \int_{\Omega}\left|u_{j}\right|\left|u_{j}-u\right| d x\right) \\
& \leq \limsup _{j \rightarrow \infty}\left(\int_{\Omega} u_{j}^{6} d x\right)^{\frac{5}{6}}\left(\int_{\Omega}\left|u_{j}-u\right|^{6} d x\right)^{\frac{1}{6}} \\
& \quad+C_{\delta} \limsup _{j \rightarrow \infty}\left(\int_{\Omega} u_{j}^{2} d x\right)^{\frac{1}{2}}\left(\int_{\Omega}\left|u_{j}-u\right|^{2} d x\right)^{\frac{1}{2}} \\
& \leq C \delta
\end{aligned}
$$

for all $\delta>0$, where for the last inequality we use the Sobolev embedding and the boundedness and $L^{2}(\Omega)$ convergence of $\left\{u_{j}\right\}$. This proves (2.12). Next we show (2.13). Actually, by the Hölder inequality, the boundedness of $\left\{u_{j}\right\}$ and the $L^{6}(\Omega)$ convergence of $\left(u_{j}\right)+$ we get

$$
\begin{aligned}
\limsup _{j \rightarrow \infty}\left|\int_{\Omega}\left(u_{j}\right)_{+}^{5}\left(u_{j}-u\right) d x\right| \leq & \limsup _{j \rightarrow \infty}\left(\int_{\Omega}\left(u_{j}\right)_{+}^{6} d x\right)^{\frac{5}{6}} \\
& \times\left(\int_{\Omega}\left|\left(u_{j}\right)_{+}-u\right|^{6} d x\right)^{\frac{1}{6}} \\
= & 0,
\end{aligned}
$$

here we use the relation $\left(u_{j}\right)_{+}^{5} u_{j}=\left(u_{j}\right)_{+}^{6}$. This shows (2.13). Consequently from (2.11)-(2.13), we conclude that

$$
\left(a+b\left\|u_{j}\right\|^{2}\right) \int_{\Omega} \nabla u_{j} \cdot \nabla\left(u_{j}-u\right) d x=o(1) \quad \text { as } j \rightarrow \infty .
$$

By the weak convergence, we have $\left\|u_{j}\right\| \rightarrow\|u\|$ as $j \rightarrow \infty$. This completes the proof.

Here we recall $(\mathrm{g} 4)$ :

(g4) There exists a nonempty open set $\omega \subset \Omega$ such that

$$
\lim _{u \rightarrow \infty} \frac{g(x, u)}{u^{3}}=\infty
$$

uniformly for $x \in \omega$.

With no loss of generality, we can assume $0 \in \omega$. For every $\varepsilon>0$, we define a cut off Talenti function in $\Omega$ so that

$$
u_{\varepsilon}(x)=\frac{\varepsilon^{\frac{1}{2}} \tau(x)}{\left(\varepsilon^{2}+|x|^{2}\right)^{\frac{1}{2}}}
$$


where $\tau$ is a smooth function in $\Omega$ such that $\tau=1$ on some neighborhood of 0 and $\operatorname{spt} \tau \subset \omega$. From the estimate in [9], we get

$$
\left\{\begin{array}{l}
\int_{\Omega}\left|\nabla u_{\varepsilon}\right|^{2} d x=K_{1}+O(\varepsilon) \\
\int_{\Omega} u_{\varepsilon}^{6} d x=K_{2}^{3}+O\left(\varepsilon^{2}\right) \\
\int_{\Omega} u_{\varepsilon}^{2} d x=O(\varepsilon)
\end{array}\right.
$$

where $K_{1}, K_{2}>0$ are some constants with $S=K_{1} / K_{2}$. Here $S>0$ is given by

$$
S:=\inf _{u \in H_{0}^{1}(\Omega) \backslash\{0\}} \frac{\int_{\Omega}|\nabla u|^{2} d x}{\left(\int_{\Omega} u^{6} d x\right)^{\frac{1}{3}}} .
$$

Set

$$
v_{\varepsilon}(x):=\frac{u_{\varepsilon}(x)}{\left(\int_{\Omega} u_{\varepsilon}^{6} d x\right)^{\frac{1}{6}}} .
$$

Then by (2.14), we have

$$
\left\{\begin{array}{l}
\int_{\Omega}\left|\nabla v_{\varepsilon}\right|^{2} d x=S+O(\varepsilon) \\
\int_{\Omega} v_{\varepsilon}^{6} d x=1 \\
\int_{\Omega} v_{\varepsilon}^{2} d x=O(\varepsilon)
\end{array}\right.
$$

The following lemma gives a sufficient condition on $g$ (which is weaker than (g4)) to ensure the compactness of $(\mathrm{PS})_{c}$ sequences for $I$. The argument is strictly based on Lemma 2.1 in [9].

Lemma 2.6. Let g satisfy (g1) and (g2). We assume that there exist a nonempty open set $\omega \subset \Omega$ and a measurable function $g(u)$ such that $g(x, u) \geq g(u) \geq 0$ for all $x \in \omega$ and $u \geq 0$ and further,

$$
\lim _{\varepsilon \rightarrow 0} \varepsilon^{2} \int_{0}^{\varepsilon^{-1}} G\left(\frac{\varepsilon^{-\frac{1}{2}}}{\left(1+s^{2}\right)^{\frac{1}{2}}}\right) s^{2} d s=\infty
$$

where $G(t):=\int_{0}^{t} g(s) d s$, then there exists a constant $\varepsilon_{0}>0$ such that

$$
\max _{t \geq 0} I\left(t v_{\varepsilon}\right)<\frac{a}{2} C_{K}+\frac{b}{4} C_{K}^{2}-\frac{1}{6 S^{3}} C_{K}^{3}
$$

for all $\varepsilon \in\left(0, \varepsilon_{0}\right)$.

Proof. We define $v_{\varepsilon}$ as above. Take $t \geq 0$. By (2.15), we have

$$
I\left(t v_{\varepsilon}\right)=\frac{a t^{2}}{2}\left\|v_{\varepsilon}\right\|^{2}+\frac{b t^{4}}{4}\left\|v_{\varepsilon}\right\|^{4}-\mu \int_{\Omega} G\left(x, t v_{\varepsilon}\right) d x-\frac{t^{6}}{6}=: f(t) .
$$

We take $t_{\varepsilon}$ so that $f\left(t_{\varepsilon}\right)=\max _{t \geq 0} f(t)$. From Lemma 2.1, clearly we have $t_{\varepsilon}>0$. Put $A_{\varepsilon}:=\left\|v_{\varepsilon}\right\|^{2}$. Then noting (g1) and (g2), we get

$$
a A_{\varepsilon}+b t_{\varepsilon}^{2} A_{\varepsilon}^{2}-\mu \int_{\Omega} \frac{g\left(x, t_{\varepsilon} v_{\varepsilon}\right) v_{\varepsilon}}{t_{\varepsilon}} d x-t_{\varepsilon}^{4}=0 .
$$

Since $g(x, u) \geq 0$ for all $x \in \Omega$ and $u \geq 0$ by (g1), we have

$$
t_{\varepsilon} \leq\left\{\frac{1}{2}\left(b A_{\varepsilon}^{2}+\sqrt{\left(b A_{\varepsilon}^{2}\right)^{2}+4 a A_{\varepsilon}}\right)\right\}^{\frac{1}{2}}=: T_{\varepsilon} .
$$


Since the map

$$
t \mapsto \frac{a t^{2}}{2} A_{\varepsilon}+\frac{b t^{4}}{4} A_{\varepsilon}^{2}-\frac{t^{6}}{6}
$$

is increasing on the interval $\left[0, T_{\varepsilon}\right]$, we have

$$
\begin{aligned}
I\left(t v_{\varepsilon}\right) & \leq I\left(t_{\varepsilon} v_{\varepsilon}\right) \\
& \leq \frac{a T_{\varepsilon}^{2}}{2} A_{\varepsilon}+\frac{b T_{\varepsilon}^{4}}{4} A_{\varepsilon}^{2}-\frac{T_{\varepsilon}^{6}}{6}-\mu \int_{\Omega} G\left(x, t_{\varepsilon} v_{\varepsilon}\right) d x \\
& \leq \frac{a}{2} C_{K}+\frac{b}{4} C_{K}^{2}-\frac{1}{6 S^{3}} C_{K}^{3}+O(\varepsilon)-\mu \int_{\Omega} G\left(x, t_{\varepsilon} v_{\varepsilon}\right) d x
\end{aligned}
$$

after some calculation. Hence if

$$
\lim _{\varepsilon \rightarrow 0} \varepsilon^{-1} \int_{\Omega} G\left(x, t_{\varepsilon} v_{\varepsilon}\right) d x=\infty
$$

we can take $\varepsilon_{0}>0$ so small that

$$
\sup _{t \geq 0} I\left(t v_{\varepsilon}\right)<\frac{a}{2} C_{K}+\frac{b}{4} C_{K}^{2}-\frac{1}{6 S^{3}} C_{K}^{3}
$$

for all $\varepsilon \in\left(0, \varepsilon_{0}\right)$. This finishes the proof. Now we show (2.18). To this end, first notice that

$$
t_{\varepsilon} \rightarrow\left\{\frac{1}{2}\left(b S^{4}+\sqrt{\left(b S^{4}\right)^{2}+4 a S^{2}}\right)\right\}^{\frac{1}{2}} .
$$

(2.19) comes from the fact that

$$
\int_{\Omega} \frac{g\left(x, t_{\varepsilon} v_{\varepsilon}\right) v_{\varepsilon}}{t_{\varepsilon}} d x \rightarrow 0
$$

as $\varepsilon \rightarrow 0$. In fact, by (2.1), we get

$$
\int_{\Omega} \frac{g\left(x, t_{\varepsilon} v_{\varepsilon}\right) v_{\varepsilon}}{t_{\varepsilon}} d x \leq \delta t_{\varepsilon}^{4}+C_{\delta} \int_{\Omega} v_{\varepsilon}^{2} d x
$$

for all $\delta>0$ and some constant $C_{\delta}>0$. On the other hand, since $T_{\varepsilon}$ is bounded, $t_{\varepsilon}$ is also bounded by (2.17). Using this fact and (2.15), we have

$$
\limsup _{\varepsilon \rightarrow 0} \int_{\Omega} \frac{g\left(x, t_{\varepsilon} v_{\varepsilon}\right) v_{\varepsilon}}{t_{\varepsilon}} d x \leq C \delta
$$

for some constant $C>0$ and all $\delta>0$. This ensures (2.20). After this, the argument is completely same as that in the proof of Lemma 2.1 in [9]. But we shall proceed with the rest for reader's convenience. By the assumption on $g$, recalling (2.19) and (2.15), we have 


$$
\begin{aligned}
\varepsilon^{-1} \int_{\Omega} G\left(x, t_{\varepsilon} v_{\varepsilon}\right) d x & \geq \varepsilon^{-1} \int_{\omega} G\left(t_{\varepsilon} v_{\varepsilon}\right) d x \\
& \geq \varepsilon^{-1} \int_{\omega} G\left(C_{\varepsilon} \frac{\varepsilon^{\frac{1}{2}} \tau(x)}{\left(\varepsilon^{2}+|x|^{2}\right)^{\frac{1}{2}}}\right) d x \\
& \geq C \varepsilon^{-1} \int_{0}^{r} G\left(C_{\varepsilon} \frac{\varepsilon^{\frac{1}{2}}}{\left(\varepsilon^{2}+s^{2}\right)^{\frac{1}{2}}}\right) s^{2} d s \\
& \geq C \varepsilon^{2} \int_{0}^{\frac{r}{\varepsilon}} G\left(C_{\varepsilon} \frac{\varepsilon^{-\frac{1}{2}}}{\left(1+s^{2}\right)^{\frac{1}{2}}}\right) s^{2} d s \\
& \geq C \varepsilon^{2} \int_{0}^{\frac{C}{\varepsilon}} G\left(\frac{\varepsilon^{-\frac{1}{2}}}{\left(1+s^{2}\right)^{\frac{1}{2}}}\right) s^{2} d s .
\end{aligned}
$$

for some constant $C>0$ where $C_{\varepsilon}$ denotes some constant which converges some positive value as $\varepsilon \rightarrow 0$ and for the last inequality we perform an appropriate rescaling for $\varepsilon$. If $C \geq 1,(2.18)$ immediately follows. If $C<1$, we have

$$
\begin{aligned}
\varepsilon^{2} \int_{0}^{\frac{C}{\varepsilon}} G\left(\frac{\varepsilon^{-\frac{1}{2}}}{\left(1+s^{2}\right)^{\frac{1}{2}}}\right) s^{2} d s= & \varepsilon^{2} \int_{0}^{\frac{1}{\varepsilon}} G\left(\frac{\varepsilon^{-\frac{1}{2}}}{\left(1+s^{2}\right)^{\frac{1}{2}}}\right) s^{2} d s \\
& -\varepsilon^{2} \int_{\frac{C}{\varepsilon}}^{\frac{1}{\varepsilon}} G\left(\frac{\varepsilon^{-\frac{1}{2}}}{\left(1+s^{2}\right)^{\frac{1}{2}}}\right) s^{2} d s .
\end{aligned}
$$

Put

$$
B_{\varepsilon}:=\varepsilon^{2} \int_{\frac{C}{\varepsilon}}^{\frac{1}{\varepsilon}} G\left(\frac{\varepsilon^{-\frac{1}{2}}}{\left(1+s^{2}\right)^{\frac{1}{2}}}\right) s^{2} d s .
$$

Using (2.1), we get for some $\delta>0$,

$$
\begin{aligned}
\left|B_{\varepsilon}\right| & \leq \varepsilon^{2}\left(\delta \int_{\frac{C}{\varepsilon}}^{\frac{1}{\varepsilon}}\left|\frac{\varepsilon^{-\frac{1}{2}}}{\left(1+s^{2}\right)^{\frac{1}{2}}}\right|^{6} s^{2} d s+C_{\delta} \int_{\frac{C}{\varepsilon}}^{\frac{1}{\varepsilon}}\left|\frac{\varepsilon^{-\frac{1}{2}}}{\left(1+s^{2}\right)^{\frac{1}{2}}}\right|^{2} s^{2} d s\right) \\
& \leq C
\end{aligned}
$$

for some constant $C>0$. This concludes (2.18).

We can also conclude the following lemma as in the proof of Corollary 2.3 in $[9]$.

Lemma 2.7. Suppose $g$ satisfies (g1), (g2) and (g4). Then $g$ satisfies the hypotheses of Lemma 2.6.

Proof. Set $g(u):=\inf _{x \in \omega} g(x, u)$. By $(\mathrm{g} 4)$, for all $M>0$, there exists a constant $R>0$ such that $G(u) \geq M u^{4}$ for all $u \geq R$ here $G(t):=\int_{0}^{t} g(s) d s$. We note that there exists a constant $C>0$ which is independent of $\varepsilon>0$ such that

$$
\frac{\varepsilon^{-\frac{1}{2}}}{\left(1+s^{2}\right)^{\frac{1}{2}}} \geq R
$$


for all $s \leq C \varepsilon^{-\frac{1}{2}}$ if $\varepsilon>0$ is sufficiently small. Using this fact, we get

$$
\begin{aligned}
\varepsilon^{2} \int_{0}^{\varepsilon^{-1}} G\left(\frac{\varepsilon^{-\frac{1}{2}}}{\left(1+s^{2}\right)^{\frac{1}{2}}}\right) s^{2} d s & \geq \varepsilon^{2} \int_{0}^{C \varepsilon^{-\frac{1}{2}}} G\left(\frac{\varepsilon^{-\frac{1}{2}}}{\left(1+s^{2}\right)^{\frac{1}{2}}}\right) s^{2} d s \\
& \geq M \int_{0}^{C \varepsilon^{-\frac{1}{2}}} \frac{s^{2}}{\left(1+s^{2}\right)^{2}} d s
\end{aligned}
$$

for all $M>0$ and small $\varepsilon>0$. Hence we have

$$
\liminf _{\varepsilon \rightarrow 0}\left\{\varepsilon^{2} \int_{0}^{\varepsilon^{-1}} G\left(\frac{\varepsilon^{-\frac{1}{2}}}{\left(1+s^{2}\right)^{\frac{1}{2}}}\right) s^{2} d s\right\} \geq C M
$$

for all $M>0$ and some constant $C>0$ which is independent of $M>0$. This concludes the proof.

Finally we prove Theorem 1.1.

Proof of Theorem 1.1. Let $g$ satisfy (g1)-(g4). Define the mountain pass energy $c$ as below Lemma 2.2. Then as stated before, there exists a $(\mathrm{PS})_{c}$ sequence. On the other hand, from Lemma 2.2 and the definition of $c$, for any $\varepsilon>0$, we have a constant $t_{0}>0$ such that $\gamma(t):=t\left(t_{0} v_{\varepsilon}\right) \in \Gamma$. Thus from Lemma 2.5, Lemma 2.6 and Lemma 2.7, I satisfies the $(\mathrm{PS})_{c}$ condition. Hence we conclude that $I$ has a nontrivial critical point $u \in H_{0}^{1}(\Omega)$ with its critical value $c \geq \alpha$. Furthermore, similarly to Remark 2.4 , we can show that $u \in H_{0}^{1}(\Omega)$ is nonnegative. The smoothness and positivity of $u$ is shown as we note in the end of Sect. 1. This finishes the proof.

Proof of Corollary 1.2. We assume $4<q<6$ and $g(x, u):=u^{q}$. For the proof, we consider instead $g(x, u):=\left(u_{+}\right)^{q}$. Then clearly $g$ satisfies $(\mathrm{g} 1)-(\mathrm{g} 4)$. By Theorem 1.1, we conclude the proof.

\section{Proof of Theorem 1.3}

In Sect. 3, we prove Theorem 1.3 and Corollary 1.4. Assume $a>0$ and $b \geq 0$. In addition, let $g$ satisfy (g1), (g2), (g5) and (g6). But if there exists a constant $4<\theta<6$ such that $g(x, u) u-\theta G(u) \geq 0$ for all $x \in \Omega$ and $t \geq 0$, the proof is simpler. We can refer to the argument in [2] for that situation. Hence we only consider the following condition instead of (g5):

(g5)' There exists a constant $\theta>0$ such that $2<\theta \leq 4$ and $g(x, u) u-$ $\theta G(x, u) \geq 0$ for all $x \in \Omega$ and $u \geq 0$.

As in Sect. 2, we define the energy functional associated to (K1) so that

$$
I_{\mu}(u)=\frac{a}{2}\|u\|^{2}+\frac{b}{4}\|u\|^{4}-\mu \int_{\Omega} G(x, u) d x-\frac{1}{6} \int_{\Omega} u_{+}^{6} d x,
$$

where $G(x, u):=\int_{0}^{u} g(x, t) d t$ and $u_{+}:=\max \{u, 0\}$. Inspired by $[6,20]$, we introduce a truncation method, already used in earlier researches [17,18]. Let $\psi$ be a smooth function on $[0, \infty)$ such that $\psi=1$ on $[0,1), \psi=0$ on $[2, \infty)$ 
and $0 \leq \psi \leq 1$ otherwise. Furthermore, we assume $-2 \leq \psi^{\prime} \leq 0$ on $[0, \infty)$. For $T>0$, define a $C^{1}$ cut off functional $\Phi_{T}(u)$ on $H_{0}^{1}(\Omega)$ so that

$$
\Phi_{T}(u):=\psi\left(\frac{\|u\|^{2}}{T^{2}}\right)
$$

We consider a truncated functional on $H_{0}^{1}(\Omega)$ such that

$$
J_{\mu}^{T}(u)=\frac{a}{2}\|u\|^{2}+\frac{b}{4}\|u\|^{4} \Phi_{T}(u)-\mu \int_{\Omega} G(x, u) d x-\frac{1}{6} \int_{\Omega} u_{+}^{6} d x .
$$

Then by (g1) and (g2), we can easily verify that $J_{\lambda}^{T}$ is well defined and continuously Fréchet differentiable on $H_{0}^{1}(\Omega)$. Its first Fréchet derivative is given by

$$
\begin{aligned}
\left\langle J_{\mu}^{T^{\prime}}(u), h\right\rangle= & \left\{\left(a+b\|u\|^{2} \Phi_{T}(u)+\frac{b}{2 T^{2}}\|u\|^{4} \psi^{\prime}\left(\frac{\|u\|^{2}}{T^{2}}\right)\right\} \int_{\Omega} \nabla u \cdot \nabla h d x\right. \\
& -\mu \int_{\Omega} g(x, u) h d x-\int_{\Omega} u_{+}^{5} h d x \quad\left(h \in H_{0}^{1}(\Omega)\right) .
\end{aligned}
$$

Choose $T>0$ as

$$
T=\min \left\{\left(\frac{a}{8 b}\right)^{\frac{1}{2}},\left\{\frac{a(\theta-2)}{4 b(4-\theta)}\right\}^{\frac{1}{2}}\right\} .
$$

Note that by (3.1) and the fact that $\left|\|u\|^{4} \psi^{\prime}\left(\|u\|^{2} / T^{2}\right)\right| \leq 8 T^{4}$ for all $u \in$ $H_{0}^{1}(\Omega)$, the following relations hold.

$$
\begin{gathered}
a+\frac{b}{2 T^{2}}\|u\|^{4} \psi^{\prime}\left(\frac{\|u\|^{2}}{T^{2}}\right) \geq \frac{a}{2}, \\
a\left(\frac{1}{2}-\frac{1}{\theta}\right)-2 b\left(\frac{1}{\theta}-\frac{1}{4}\right) T^{2} \geq 0,
\end{gathered}
$$

and

$$
8 b\left(\frac{1}{\theta}-\frac{1}{4}\right) T^{4} \leq a\left(\frac{1}{2}-\frac{1}{\theta}\right) T^{2} .
$$

Note also that if $u \in H_{0}^{1}(\Omega)$ and $\|u\|<T, J_{\mu}^{T}(u)=I_{\mu}(u)$ on some neighborhood of $u$. Thus if $u$ is a critical point of $J_{\mu}^{T}$ with $\|u\|<T, u$ is also a critical point of $I_{\mu}$. From now on, we show the existence of a nontrivial critical point $u$ of $J_{\mu}^{T}$ with $\|u\|<T$. Since we fix $T>0$ as in (3.1), we denote $J_{\mu}^{T}(u)$ and $\Phi_{T}(u)$ as $J_{\mu}(u)$ and $\Phi(u)$ respectively for simplicity.

We begin with following two lemmas.

Lemma 3.1. We assume $g$ satisfies (g1) and (g2). Then there exist constants $\alpha, \rho>0$ such that $J_{\mu}(u) \geq \alpha$ for all $u \in H_{0}^{1}(\Omega)$ with $\|u\|=\rho$.

Proof. For $\rho>0$, take $u \in H_{0}^{1}(\Omega)$ with $\|u\|=\rho$. By (g1) and (g2), as in the proof of Lemma 2.1, we get the inequality

$$
J_{\mu}(u) \geq \frac{a}{4}\|u\|^{2}-C\|u\|^{6}=\frac{a}{4} \rho^{2}-C \rho^{6}
$$


for some constant $C>0$. Hence by taking $\rho$ small enough, we conclude that there exists a constant $\alpha>0$ such that

$$
J_{\mu}(u) \geq \alpha .
$$

This finishes the proof.

Lemma 3.2. Suppose $g$ satisfies (g1) and (g2). Then there exists a function $v_{0} \in H_{0}^{1}(\Omega)$ such that $\left\|v_{0}\right\|>\rho$ and $J_{\mu}\left(v_{0}\right) \leq 0$.

Proof. Take a nontrivial function $v \in H_{0}^{1}(\Omega)$ with $v \geq 0$ and $t>0$. Since by (g1), $G(x, s) \geq 0$ for all $x \in \Omega$ and $s \in \mathbb{R}$, we get

$$
J_{\mu}(t v) \leq \frac{a t^{2}}{2}\|v\|^{2}+\frac{b t^{4}}{4}\|v\|^{4}-\frac{t^{6}}{6} \int_{\Omega} v^{6} d x .
$$

Thus we have $J_{\mu}(t v) \rightarrow-\infty$ as $t \rightarrow \infty$. Hence there exists a constant $t_{0}>0$ such that if $v_{0}:=t_{0} v,\left\|v_{0}\right\|>\rho$ and $J_{\mu}\left(v_{0}\right) \leq 0$. This concludes the proof.

As in Sect. 2 we define

$$
\Gamma:=\left\{\gamma \in C\left([0,1], H_{0}^{1}(\Omega)\right) \mid \gamma(0)=0, J_{\mu}(\gamma(1)) \leq 0, \gamma(1) \neq 0\right\}
$$

and

$$
c_{\mu}:=\inf _{\gamma \in \Gamma} \max _{u \in \gamma([0,1])} J_{\mu}(u) .
$$

We prove the next lemma.

Lemma 3.3. Let $g$ satisfy (g1), (g2) and (g6). Then $c_{\mu} \rightarrow 0$ as $\mu \rightarrow \infty$.

Proof. As in the proof of corollary 2.4 in [9], we assume $0 \in \omega$ and take a function $\xi \in C_{0}^{\infty}(\omega)$ with $\xi(0)=1$ and a constant $0<k<1 / 2$. Set $v:=\xi|x|^{-k}$. Then $v \in H_{0}^{1}(\Omega)$ by a null extension. By the definition of $v$ and (g1), we have

$$
J_{\mu}(t v) \leq \frac{a t^{2}}{2}\|v\|^{2}+\frac{b t^{4}}{4}\|v\|^{4}-\mu \int_{\omega} G(x, t v) d x-\frac{t^{6}}{6} \int_{\Omega} v^{6} d x=: f(t) .
$$

We put $t_{\mu}>0$ so that $f\left(t_{\mu}\right)=\max _{t \geq 0} f(t)$. Then, using (g2) we have

$$
a\|v\|^{2}+b t_{\mu}^{2}\|v\|^{4}-\frac{\mu}{t_{\mu}} \int_{\omega} g\left(x, t_{\mu} v\right) v d x-t_{\mu}^{4} \int_{\Omega} v^{6}=0 .
$$

By (g1), we get

$$
a\|v\|^{2}+b t_{\mu}^{2}\|v\|^{4} \geq t_{\mu}^{4} \int_{\Omega} v^{6} .
$$

Hence there exists a constant $C>0$ such that $t_{\mu} \leq C$ for all $\mu>0$. Furthermore $t_{\mu} \rightarrow 0$ as $\mu \rightarrow \infty$. If not, there exist a sequence $\left\{\mu_{n}\right\}$ and a constant $\beta>0$ such that $\mu_{n} \rightarrow \infty$ and $t_{\mu_{n}} \rightarrow \beta$ as $n \rightarrow \infty$. Then by (g1) and (g2), we have

$$
\frac{1}{t_{\mu_{n}}} \int_{\omega} g\left(x, t_{\mu_{n}} v\right) v d x \rightarrow \frac{1}{\beta} \int_{\omega} g(x, \beta v) v d x>0
$$


The positivity of the right hand side is ensured by (g6) and the definition of $v$. But in view of $(3.5), \int_{\omega} g(x, \beta v) v d x$ must be 0 . This is a contradiction. Therefore we obtain

$$
0<c_{\mu} \leq \max _{t \geq 0} J_{\mu}(t v) \leq \max _{t \geq 0} f(t)<\frac{a t_{\mu}^{2}}{2}\|v\|^{2}+\frac{b t_{\mu}^{4}}{4}\|v\|^{4} \rightarrow 0 .
$$

This completes the proof.

Next we prove an important lemma which ensures the local compactness of the PS sequences for $J_{\mu}$.

Lemma 3.4. We assume $g$ satisfies (g1), (g2) and (g5)' and further, $\left\{u_{j}\right\}$ is a $(P S)_{c}$ sequence for $J_{\mu}$ with

$$
c<\left(\frac{1}{\theta}-\frac{1}{6}\right)\left(\frac{a S}{2}\right)^{\frac{3}{2}} .
$$

Then $\left\{\left(u_{j}\right)_{+}\right\}$has a subsequence which strongly converges in $L^{6}(\Omega)$.

Proof. Let $\left\{u_{j}\right\} \subset H_{0}^{1}(\Omega)$ be a $(\mathrm{PS})_{c}$ sequence for $J_{\mu}$ with

$$
c<\left(\frac{1}{\theta}-\frac{1}{6}\right)\left(\frac{a S}{2}\right)^{\frac{3}{2}} .
$$

Firstly we claim that $\left\{u_{j}\right\}$ is bounded in $H_{0}^{1}(\Omega)$. Actually, since $J_{\mu}\left(u_{j}\right) \rightarrow c$ and $J_{\mu}^{\prime}\left(u_{j}\right) \rightarrow 0$ in $H^{-1}(\Omega)$, we have by (g1) and (g5)',

$$
\begin{aligned}
c+1 \geq & J_{\mu}\left(u_{j}\right)-\frac{1}{\theta}\left\langle J_{\mu}^{\prime}\left(u_{j}\right), u_{j}\right\rangle+\frac{1}{\theta}\left\langle J_{\mu}^{\prime}\left(u_{j}\right), u_{j}\right\rangle \\
\geq & a\left(\frac{1}{2}-\frac{1}{\theta}\right)\left\|u_{j}\right\|^{2}-b\left(\frac{1}{\theta}-\frac{1}{4}\right)\left\|u_{j}\right\|^{4} \Phi(u)-\frac{b}{2 \theta T^{2}}\left\|u_{j}\right\|^{6} \psi^{\prime}\left(\frac{\left\|u_{j}\right\|^{2}}{T^{2}}\right) \\
& \quad+\mu \int_{\Omega}\left(\frac{1}{\theta} g\left(x, u_{j}\right)-G\left(x, u_{j}\right)\right) d x+\left(\frac{1}{\theta}-\frac{1}{6}\right) \int_{\Omega}\left(u_{j}\right)_{+}^{6} d x-\left\|u_{j}\right\| \\
\geq & a\left(\frac{1}{2}-\frac{1}{\theta}\right)\left\|u_{j}\right\|^{2}-4 b\left(\frac{1}{\theta}-\frac{1}{4}\right) T^{4}-\left\|u_{j}\right\|,
\end{aligned}
$$

for large $j \in \mathbb{N}$, where for the last inequality we use the facts that $\|u\|^{4} \Phi(u) \leq$ $4 T^{4}$ for all $u \in H_{0}^{1}(\Omega)$ and $\psi^{\prime} \leq 0$ on $[0, \infty)$. This inequality proves the claim. Consequently as in the previous section, we conclude that there exists a function $u \in H_{0}^{1}(\Omega)$ such that

$$
\begin{aligned}
& u_{j} \rightarrow u \text { weakly in } H_{0}^{1}(\Omega), \\
& u_{j} \rightarrow u \text { in } L^{p}(\Omega) \text { for all } 1 \leq p<6, \\
& u_{j} \rightarrow u \text { a.e. on } \Omega,
\end{aligned}
$$

up to subsequences but still denoted $\left\{u_{j}\right\}$. Furthermore by the second concentration compactness lemma, there exist an at most countable set $\mathcal{J}$, points $\left(x_{k}\right)_{k \in \mathcal{J}} \subset \bar{\Omega}$ and positive values $\left(\eta_{k}\right)_{k \in \mathcal{J}},\left(\nu_{k}\right)_{k \in \mathcal{J}}$ with

$$
\eta_{k} \geq S \nu_{k}^{\frac{1}{3}}(k \in \mathcal{J})
$$


such that

$$
\begin{gathered}
\left|\nabla u_{j}\right|^{2} \rightarrow d \eta \geq|\nabla u|^{2}+\sum_{k \in \mathcal{J}} \eta_{k} \delta_{x_{k}}, \\
\left(u_{j}\right)_{+}^{6} \rightarrow d \nu=u_{+}^{6}+\sum_{k \in \mathcal{J}} \nu_{k} \delta_{x_{k}}
\end{gathered}
$$

in the measure sense. To complete the proof, we claim $\mathcal{J}=\emptyset$. If not, choose $k \in \mathcal{J}$. We define a test function $\phi \in C_{0}^{\infty}\left(\mathbb{R}^{3}\right)$ such that $\phi=1$ on $B\left(x_{k}, \varepsilon\right)$, $\phi=0$ on $B\left(x_{k}, 2 \varepsilon\right)^{c}$, and $0 \leq \phi \leq 1$ otherwise. In addition we assume $|\nabla \phi| \leq$ $2 / \varepsilon$. Then we have

$$
\begin{aligned}
0= & \lim _{j \rightarrow \infty}\left\langle J_{\mu}^{\prime}\left(u_{j}\right), u_{j} \phi\right\rangle \\
= & \lim _{j \rightarrow \infty}\left\{a+b\left\|u_{j}\right\|^{2} \Phi\left(u_{j}\right)+\frac{b}{2 T^{2}}\left\|u_{j}\right\|^{4} \psi^{\prime}\left(\frac{\left\|u_{j}\right\|^{2}}{T^{2}}\right)\right\} \int_{\Omega} \nabla u_{j} \cdot \nabla\left(u_{j} \phi\right) d x \\
& -\mu \lim _{j \rightarrow \infty} \int_{\Omega} g\left(x, u_{j}\right) u_{j} \phi d x-\lim _{j \rightarrow \infty} \int_{\Omega}\left(u_{j}\right)_{+}^{6} \phi d x .
\end{aligned}
$$

Now we estimate the first and second terms in the right hand side of (3.6). We compute the first term so that

$$
\begin{aligned}
\lim _{j \rightarrow \infty} & \left\{a+b\left\|u_{j}\right\|^{2} \Phi\left(u_{j}\right)+\frac{b}{2 T^{2}}\left\|u_{j}\right\|^{4} \psi^{\prime}\left(\frac{\left\|u_{j}\right\|^{2}}{T^{2}}\right)\right\} \int_{\Omega} \nabla u_{j} \cdot \nabla\left(u_{j} \phi\right) d x \\
= & \lim _{j \rightarrow \infty}\left\{a+b\left\|u_{j}\right\|^{2} \Phi\left(u_{j}\right)+\frac{b}{2 T^{2}}\left\|u_{j}\right\|^{4} \psi^{\prime}\left(\frac{\left\|u_{j}\right\|^{2}}{T^{2}}\right)\right\} \int_{\Omega}\left|\nabla u_{j}\right|^{2} \phi d x \\
& +\lim _{j \rightarrow \infty}\left\{a+b\left\|u_{j}\right\|^{2} \Phi\left(u_{j}\right)+\frac{b}{2 T^{2}}\left\|u_{j}\right\|^{4} \psi^{\prime}\left(\frac{\left\|u_{j}\right\|^{2}}{T^{2}}\right)\right\} \int_{\Omega}\left(\nabla u_{j} \cdot \nabla \phi\right) u_{j} d x \\
\geq & \lim _{j \rightarrow \infty} \frac{a}{2} \int_{\Omega}\left|\nabla u_{j}\right|^{2} \phi d x+o(1),
\end{aligned}
$$

where $o(1) \rightarrow 0$ as $\varepsilon \rightarrow 0$. The last inequality comes from (3.2) and the fact that

$$
\begin{aligned}
& \lim _{j \rightarrow \infty}\left|\left\{a+b\left\|u_{j}\right\|^{2} \Phi\left(u_{j}\right)+\frac{b}{2 T^{2}}\left\|u_{j}\right\|^{4} \psi^{\prime}\left(\frac{\left\|u_{j}\right\|^{2}}{T^{2}}\right)\right\} \int_{\Omega}\left(\nabla u_{j} \cdot \nabla \phi\right) u_{j} d x\right| \\
& \quad=o(1) \quad \text { as } \varepsilon \rightarrow 0 .
\end{aligned}
$$

We verify (3.8). Using the facts $\|u\|^{2} \Phi(u) \leq 2 T^{2}$ and \|\|$u_{j} \|^{4} \psi^{\prime}\left(\left\|u_{j}\right\|^{2} / T^{2}\right) \mid \leq$ $8 T^{4}$ for all $u \in H_{0}^{1}(\Omega)$ we estimate

$$
\begin{aligned}
& \lim _{j \rightarrow \infty}\left|\left(a+b\left\|u_{j}\right\|^{2} \Phi\left(u_{j}\right)+\frac{b}{2 T^{2}}\left\|u_{j}\right\|^{4} \psi^{\prime}\left(\frac{\left\|u_{j}\right\|^{2}}{T^{2}}\right)\right) \int_{\Omega} \nabla u_{j} \cdot \nabla \phi u_{j} d x\right| \\
& \leq\left(a+6 b T^{2}\right) \lim _{j \rightarrow \infty} \int_{\Omega \cap B\left(x_{k}, 2 \varepsilon\right)}\left|\nabla u_{j} \| u_{j} \nabla \phi\right| d x \\
& \leq\left(a+6 b T^{2}\right) \lim _{j \rightarrow \infty}\left\{\left(\int_{\Omega}\left|\nabla u_{j}\right|^{2} d x\right)^{\frac{1}{2}}\left(\int_{\Omega \cap B\left(x_{k}, 2 \varepsilon\right)}\left|u_{j} \nabla \phi\right|^{2} d x\right)^{\frac{1}{2}}\right\}
\end{aligned}
$$




$$
\begin{aligned}
& \leq C\left(\int_{\Omega \cap B\left(x_{k}, 2 \varepsilon\right)} u^{6} d x\right)^{\frac{1}{6}}\left(\int_{\Omega \cap B\left(x_{k}, 2 \varepsilon\right)}|\nabla \phi|^{3} d x\right)^{\frac{1}{3}} \\
& \leq C\left(\int_{\Omega \cap B\left(x_{k}, 2 \varepsilon\right)} u^{6} d x\right)^{\frac{1}{6}} \rightarrow 0 \quad \text { as } \varepsilon \rightarrow 0,
\end{aligned}
$$

where for the second inequality we use the Schwartz inequality, and for the third inequality we use the boundedness and $L^{2}(\Omega)$ convergence of $\left\{u_{j}\right\}$ and the Hölder inequality, and for the fourth inequality we use our assumption that $|\nabla \phi| \leq 2 / \varepsilon$. This proves (3.8). Next using (2.1), we compute the second term in the right hand side of (3.6) similarly to (2.7) in the previous section so that

$$
\lim _{j \rightarrow \infty} \int_{\Omega} g\left(x, u_{j}\right) u_{j} \phi d x=o(1) \quad \text { as } \varepsilon \rightarrow 0 .
$$

Using the computations (3.7) and (3.9) for (3.6), we get

$$
\begin{aligned}
0 & \geq \lim _{j \rightarrow \infty}\left(\frac{a}{2} \int_{\Omega}\left|\nabla u_{j}\right|^{2} \phi d x-\int_{\Omega}\left(u_{j}\right)_{+}^{6} \phi d x\right)+o(1) \\
& \geq \frac{a}{2} \int_{\bar{\Omega}} \phi d \eta-\int_{\bar{\Omega}} \phi d \nu+o(1) \text { as } \varepsilon \rightarrow 0 .
\end{aligned}
$$

Taking $\varepsilon \rightarrow 0$, we obtain

$$
\nu_{k} \geq \frac{a}{2} \eta_{k}
$$

Recalling that $\eta_{k} \geq S \nu_{k}^{\frac{1}{3}}$, we estimate

$$
\nu_{k} \geq\left(\frac{a S}{2}\right)^{\frac{3}{2}}
$$

Since $J_{\mu}\left(u_{j}\right) \rightarrow c$ and $J_{\mu}^{\prime}\left(u_{j}\right) \rightarrow 0$ in $H^{-1}(\Omega)$, we have by (3.3), (g1), (g5)' and the fact that $\psi^{\prime} \leq 0$,

$$
\begin{aligned}
c= & \lim _{j \rightarrow \infty}\left\{J_{\mu}\left(u_{j}\right)-\frac{1}{\theta}\left\langle J_{\mu}^{\prime}\left(u_{j}\right), u_{j}\right\rangle\right\} \\
= & \lim _{j \rightarrow \infty}\left[\left\{a\left(\frac{1}{2}-\frac{1}{\theta}\right)-b\left(\frac{1}{\theta}-\frac{1}{4}\right)\left\|u_{j}\right\|^{2} \Phi\left(u_{j}\right)\right\} \int_{\Omega}\left|\nabla u_{j}\right|^{2} d x\right] \\
& -\lim _{j \rightarrow \infty} \frac{b}{2 \theta T^{2}}\left\|u_{j}\right\|^{6} \psi^{\prime}\left(\frac{\left\|u_{j}\right\|^{2}}{T^{2}}\right)+\mu \lim _{j \rightarrow \infty} \int_{\Omega}\left(\frac{1}{\theta} g\left(u_{j}\right) u_{j}-G\left(u_{j}\right)\right) d x \\
& +\lim _{j \rightarrow \infty}\left(\frac{1}{\theta}-\frac{1}{6}\right) \int_{\Omega}\left(u_{j}\right)_{+}^{6} d x \\
\geq & \left(\frac{1}{\theta}-\frac{1}{6}\right)\left(\frac{a S}{2}\right)^{\frac{3}{2}} .
\end{aligned}
$$


This is a contradiction. Hence $\mathcal{J}=\emptyset$. Consequently we have

$$
\int_{\Omega}\left(u_{j}\right)_{+}^{6} d x \rightarrow \int_{\Omega} u_{+}^{6} d x \quad \text { as } j \rightarrow \infty,
$$

up to subsequences but still denoted $\left\{u_{j}\right\}$. This completes the proof.

Remark 3.5. As in Remark 2.4, we can show that $u$ is nonnegative. of $J_{\mu}$.

Using Lemmas 3.1-3.4 we prove the existence of a nontrivial critical point

Lemma 3.6. Let $g$ satisfies (g1), (g2), (g5)' and (g6). Then there exists a constant $\mu^{*} \geq 0$ such that $J_{\mu}$ has a nontrivial critical point for all $\mu>\mu^{*}$.

Proof. From Lemma 3.3, there exists a constant $\mu^{*} \geq 0$ such that

$$
c_{\mu}<\left(\frac{1}{\theta}-\frac{1}{6}\right)\left(\frac{a S}{2}\right)^{\frac{3}{2}}
$$

for all $\mu>\mu^{*}$. For each $\mu>\mu^{*}$, let $\left\{u_{j}\right\}$ be a $(\mathrm{PS})_{c_{\mu}}$ sequence for $J_{\mu}$. Then by Lemma 3.4 and Remark 3.5, there exists a nonnegative function $u \in H_{0}^{1}(\Omega)$ such that

$$
\left(u_{j}\right)_{+} \rightarrow u \text { in } L^{6}(\Omega)
$$

up to subsequences. From now on we prove that $J_{\mu}$ satisfies the $(\mathrm{PS})_{c_{\mu}}$ condition. Since $\left(u_{j}-u\right)$ is bounded in $H_{0}^{1}(\Omega)$, by the definition, we have

$$
\left\langle J_{\mu}^{\prime}\left(u_{j}\right), u_{j}-u\right\rangle=o(1)
$$

where $o(1) \rightarrow 0$ as $j \rightarrow \infty$. Furthermore, noting $u$ is nonnegative, similarly to (2.12) and (2.13) in Sect. 2, we have

$$
\int_{\Omega} g\left(u_{j}\right)\left(u_{j}-u\right) d x=o(1) \quad \text { as } j \rightarrow \infty,
$$

and

$$
\int_{\Omega}\left(u_{j}\right)_{+}^{5}\left(u_{j}-u\right) d x=o(1) \quad \text { as } j \rightarrow \infty .
$$

Combining (3.12)-(3.14), we get

$$
\begin{aligned}
& \left\{a+b\left\|u_{j}\right\|^{2} \Phi\left(u_{j}\right)+\frac{b}{2 T^{2}}\left\|u_{j}\right\|^{4} \psi^{\prime}\left(\frac{\left\|u_{j}\right\|^{2}}{T^{2}}\right)\right\} \int_{\Omega} \nabla u_{j} \cdot \nabla\left(u_{j}-u\right) d x \\
& \quad=o(1) \quad \text { as } j \rightarrow \infty
\end{aligned}
$$

Noting (3.2) and the weak convergence of $\left\{u_{j}\right\}$, we obtain

$$
\left\|u_{j}\right\| \rightarrow\|u\| \quad \text { as } j \rightarrow \infty \text {. }
$$

This proves the $(\mathrm{PS})_{c_{\mu}}$ condition for $J_{\mu}$. Now noting Lemma 3.1, Lemma 3.2, and the $(\mathrm{PS})_{c_{\mu}}$ conditions for $I_{\mu}$, the mountain pass theorem concludes the proof. 
Finally we prove Theorem 1.3.

Proof of Theorem 1.3. We first choose $\mu_{*} \geq 0$ so that

$$
c_{\mu}<\min \left\{\left(\frac{1}{\theta}-\frac{1}{6}\right)\left(\frac{a S}{2}\right)^{\frac{3}{2}}, 4 b\left(\frac{1}{\theta}-\frac{1}{4}\right) T^{4}\right\}
$$

for all $\mu>\mu_{*}$. Then by Lemma 3.6, for every $\mu>\mu_{*}$, there exists a nontrivial critical point $u \in H_{0}^{1}(\Omega)$ of $J_{\mu}$ with critical value $c_{\mu}$. As we have seen in the first part of this section, it is enough to show that $\|u\|<T$. In fact, since $J_{\mu}(u)=c_{\mu}$, we have

$$
\begin{aligned}
\frac{a}{2}\|u\|^{2}+\frac{b}{4}\|u\|^{4} \Phi(u) & =\mu \int_{\Omega} G(x, u) d x+\frac{1}{6} \int_{\Omega} u_{+}^{6} d x+c_{\mu} \\
& \leq \frac{\mu}{\theta} \int_{\Omega} g(x, u) u d x+\frac{1}{\theta} \int_{\Omega} u_{+}^{6} d x+c_{\mu}
\end{aligned}
$$

by (g5)'. On the other hand, since $u$ is a critical point of $J_{\mu}$, we obtain

$$
\begin{aligned}
& \frac{1}{\theta}\left\{a+b\|u\|^{2} \Phi(u)+\frac{b}{2 T^{2}}\|u\|^{4} \psi^{\prime}\left(\frac{\|u\|^{2}}{T^{2}}\right)\right\}\|u\|^{2} \\
& \quad=\frac{\mu}{\theta} \int_{\Omega} g(x, u) u d x+\frac{1}{\theta} \int_{\Omega} u_{+}^{6} d x
\end{aligned}
$$

Substituting (3.16) into (3.15), we compute

$$
\begin{aligned}
a\left(\frac{1}{2}-\frac{1}{\theta}\right)\|u\|^{2} & \leq b\left(\frac{1}{\theta}-\frac{1}{4}\right)\|u\|^{4} \Phi(u)+\frac{b}{2 \theta T^{2}}\|u\|^{6} \psi^{\prime}\left(\frac{\|u\|^{2}}{T^{2}}\right)+c_{\mu} \\
& \leq 4 b\left(\frac{1}{\theta}-\frac{1}{4}\right) T^{4}+c_{\mu}
\end{aligned}
$$

here we use the facts that $\|u\|^{4} \Phi(u) \leq 4 T^{4}$ and $\psi^{\prime} \leq 0$ on $[0, \infty)$. Now we assume $\|u\| \geq T$ to the contrary. Then we have

$$
a\left(\frac{1}{2}-\frac{1}{\theta}\right) T^{2} \leq 4 b\left(\frac{1}{\theta}-\frac{1}{4}\right) T^{4}+c_{\mu} .
$$

But from our choice of $T=T(a, b, \theta)>0$ and $\mu>\mu_{*}$, recalling (3.4), we have

$$
4 b\left(\frac{1}{\theta}-\frac{1}{4}\right) T^{4} \leq c_{\mu}<4 b\left(\frac{1}{\theta}-\frac{1}{4}\right) T^{4} .
$$

This is a contradiction. Hence $\|u\|<T$ and thus, $u$ is a critical point of $I_{\mu}$. As in the proof of Theorem 1.1, we can ensure the smoothness and the positivity of $u$. This finishes the proof.

Proof of Corollary 1.4. Considering $1<q \leq 3$ and $g(x, u):=\left(u_{+}\right)^{q}$, we can prove Corollary 1.4 by Theorem 1.3. 


\section{Additional results}

In Theorem 1.3 and Corollary 1.4, we do not consider the case $a=0, b>0$. They also do not consider such a case in [14]. Thus we naturally ask about the existence of solutions for such situation. As we shall discuss below, the situation seems to be different from the case $a>0$ and $b \geq 0$. We have partially but positive result for the existence. For our additional results, we consider the following problem. Let $1<q \leq 3$ and consider

$$
\begin{cases}-\|u\|^{2} \Delta u=\mu u^{q}+u^{5} & \text { in } \Omega, \\ u>0 & \text { in } \Omega, \\ u=0 & \text { on } \partial \Omega .\end{cases}
$$

Firstly we can prove the existence of a solution which is a local minimum of the corresponding functional of (K2) when $1<q<3$ (and also get the same conclusion when $0<q \leq 1)$. We give the following theorem.

Theorem 4.1. Let $1<q<3$. Then there exists a constant $m_{0}>0$ such that (K2) has a positive solution for all $0<\mu<m_{0}$.

We can prove Theorem 4.1 by a little modification of the argument in [33]. Thus we leave the proof for readers. How about the case $q=3$ ? For this case, we define

$$
\mu_{1}=\inf \left\{\|u\|^{4} \mid \int_{\Omega} u^{4} d x=1\right\} .
$$

We can easily check that $\mu_{1}$ is strictly positive and achieved by some function $\phi_{1}>0$ in $\Omega$. Then it is natural to consider the Kirchhoff type nonlinear eigenvalue problem:

$$
\begin{cases}-\|\phi\|^{2} \Delta \phi=\mu \phi^{3} & \text { in } \Omega \\ \phi=0 & \text { on } \partial \Omega\end{cases}
$$

In [28], they prove the existence of unbounded sequences of eigenvalues of (E). Some additional result for the problem (E) is obtained in [20]. Using the principal eigenvalue of (E) i.e. $\mu_{1}=\mu_{1}(\Omega)>0$, we state the following theorem.

Theorem 4.2. We assume $0 \in \Omega$ and there exists a constant $R>0$ such that $B(0, R) \subset \Omega$. Then if $3 \pi^{3} / 4 R<\mu<\mu_{1}(\Omega)$, there exists a solution of (K2).

Remark 4.3. Since we do not know the precise lower bound for the value $\mu_{1}=$ $\mu_{1}(\Omega)>0$ (but we can easily confirm that at least $\mu_{1}(\Omega) \geq\left\{(3 \pi)^{7} / 4^{5}\right\}^{1 / 3} / R$ if $\Omega=B(0, R)$, the set $\left\{\mu \in \mathbb{R} \mid 3 \pi^{3} / 4 R<\mu<\mu_{1}(\Omega)\right\}$ may be empty. Furthermore the necessity of the condition on $\mu>0$ seems to be difficult to prove. These are left for our future works.

In this section, we prove Theorem 4.2. As a matter of the fact, for this case we can employ the minimizing argument as in [9]. Define

$$
K:=\inf \left\{\|u\|^{4} \mid u \in H_{0}^{1}(\Omega), \int_{\Omega} u^{6} d x=1\right\}
$$


and

$$
K_{\mu}:=\inf \left\{\|u\|^{4}-\mu \int_{\Omega} u^{4} d x \mid u \in H_{0}^{1}(\Omega), \int_{\Omega} u^{6} d x=1\right\} .
$$

Notice that $K=S^{2}$ where $S$ is defined as in the previous sections. Following the argument in [9], we shall show the existence of a minimizer of $K_{\mu}$.

Lemma 4.4. If $K_{\mu}<S^{2}, K_{\mu}$ has a nontrivial and nonnegative minimizer.

Proof. We assume $K_{\mu}<S^{2}$. Let $\left\{u_{j}\right\}$ be a minimizing sequence of $K_{\mu}$. Then there holds $\int_{\Omega} u_{j}^{6} d x=1$ and

$$
\left\|u_{j}\right\|^{4}-\mu \int_{\Omega} u_{j}^{4}=K_{\mu}+o(1)
$$

where $o(1) \rightarrow 0$ as $j \rightarrow \infty$. Then from the embedding $L^{6}(\Omega) \hookrightarrow L^{4}(\Omega)$, we have that $\left\{u_{j}\right\}$ is bounded in $H_{0}^{1}(\Omega)$. Hence by the weak compactness of $H_{0}^{1}(\Omega)$ and the compactness of the Sobolev embeddings, we get

$$
\begin{aligned}
& u_{j} \rightarrow u \text { weakly in } H_{0}^{1}(\Omega), \\
& u_{j} \rightarrow u \text { in } L^{p}(\Omega) \text { for all } 1 \leq p<6, \\
& u_{j} \rightarrow u \text { a.e. on } \Omega,
\end{aligned}
$$

up to subsequences. Notice that $\left\|u_{j}\right\|^{4} \geq S^{2}$ by the definition of $\mathrm{S}$. Then from (4.1), $L^{4}(\Omega)$ convergence of $\left\{u_{j}\right\}$ and our assumption, we have

$$
\int_{\Omega} u^{4} d x \geq \frac{1}{\mu}\left(S^{2}-K_{\mu}\right)>0 .
$$

Hence $u$ is nontrivial. Now we put $v_{j}:=u_{j}-u$. Then by the weak convergence, we have

$$
\left\|u_{j}\right\|^{4}=\left\|v_{j}\right\|^{4}+\|u\|^{4}+2\left\|v_{j}\right\|^{2}\|u\|^{2}+o(1) \quad \text { as } j \rightarrow \infty .
$$

In addition by the Vitali convergence theorem,

$$
1=\int_{\Omega} u_{j}^{6} d x=\int_{\Omega} v_{j}^{6} d x+\int_{\Omega} u^{6} d x+o(1) \quad \text { as } j \rightarrow \infty .
$$

Thus we get from the definition of $S$,

$$
\begin{aligned}
1 & =\int_{\Omega} v_{j}^{6} d x+\int_{\Omega} u^{6} d x+o(1) \\
& \leq\left(\int_{\Omega} v_{j}^{6} d x\right)^{\frac{2}{3}}+\left(\int_{\Omega} u^{6} d x\right)^{\frac{2}{3}}+o(1) \\
& \leq S^{-2}\left\|v_{j}\right\|^{4}+\left(\int_{\Omega} u^{6} d x\right)^{\frac{2}{3}}+o(1)
\end{aligned}
$$

as $j \rightarrow \infty$. Now we claim

$$
\|u\|^{4}-\mu \int_{\Omega} u^{4} d x \leq K_{\mu}\left(\int_{\Omega} u^{6} d x\right)^{\frac{2}{3}} .
$$


In fact, let us first assume $K_{\mu}>0$. Then we have by (4.3) that

$$
K_{\mu} \leq \frac{K_{\mu}}{S^{2}}\left\|v_{j}\right\|^{4}+K_{\mu}\left(\int_{\Omega} u^{6} d x\right)^{\frac{2}{3}}+o(1) .
$$

Combining this inequality and (4.1), and using the relation (4.2), we have (4.4). Next if $K_{\mu} \leq 0$, we have

$$
K_{\mu} \leq K_{\mu}\left(\int_{\Omega} u^{6} d x\right)^{\frac{2}{3}}
$$

Again combining this inequality and (4.1), and using (4.2), we obtain (4.4). In addition, from above arguments we can also ensure that $v_{j}$ must converge to 0 in $H_{0}^{1}(\Omega)$. Thus we have $\int_{\Omega} u^{6} d x=1$. From this fact and (4.4), we conclude that $u$ is a nontrivial minimizer of $K_{\mu}$. Furthermore since we can trivially replace $u$ by $|u|$, we get the conclusion of Lemma (4.4).

Let us ensure the hypothesis of Lemma 4.4. We assume $0 \in \Omega$ and there exists a constant $R>0$ such that $B(0, R) \subset \Omega$. We define a cut off Talenti function $u_{\varepsilon}$ in $\Omega$ similarly to the one in Sect. 2 so that

$$
u_{\varepsilon}(x):=\frac{\varepsilon^{\frac{1}{2}} \sigma(|x|)}{\left(\varepsilon^{2}+|x|^{2}\right)^{\frac{1}{2}}},
$$

here $\sigma(r)$ is an appropriate cut off function such that $\sigma(0)=1, \sigma^{\prime}(0)=\sigma(R)=$ 0 and $\sigma(r)=0$ if $r>R$. Then we have $u_{\varepsilon} \in H_{0}^{1}(\Omega)$. We estimate as in [9],

$$
\left\{\begin{array}{l}
\int_{\Omega}\left|\nabla u_{\varepsilon}\right|^{2} d x=K_{1}+4 \pi \varepsilon \int_{0}^{R}\left|\sigma^{\prime}(r)\right|^{2} d r+O\left(\varepsilon^{2}\right), \\
\int_{\Omega} u_{\varepsilon}^{6} d x=K_{2}^{3}+O\left(\varepsilon^{2}\right), \\
\int_{\Omega} u_{\varepsilon}^{4} d x=\pi^{2}+O\left(\varepsilon^{2}\right),
\end{array}\right.
$$

where $K_{1}:=\left(3 \pi^{2}\right) / 4$ and $K_{2}:=\left(\pi^{2} / 4\right)^{1 / 3}$ with $K_{1} / K_{2}=S$. As in Sect. 2, we define

$$
v_{\varepsilon}(x)=\frac{u_{\varepsilon}(x)}{\left(\int_{\Omega} u_{\varepsilon}^{6} d x\right)^{\frac{1}{6}}}
$$

Then we get

$$
\left\{\begin{array}{l}
\int_{\Omega}\left|\nabla v_{\varepsilon}\right|^{2} d x=S+\frac{4 \pi \varepsilon}{K_{2}} \int_{0}^{R}\left|\sigma^{\prime}(r)\right|^{2} d r+O\left(\varepsilon^{2}\right) \\
\int_{\Omega} v_{\varepsilon}^{6} d x=1 \\
\int_{\Omega} v_{\varepsilon}^{4} d x=\frac{\pi^{2}}{K_{2}^{2}} \varepsilon+O\left(\varepsilon^{2}\right) .
\end{array}\right.
$$

We prove the next lemma.

Lemma 4.5. If $\mu>3 \pi^{3} / 4 R, K_{\mu}<S^{2}$.

Proof. Using (4.6), we estimate

$$
\begin{aligned}
K_{\mu} & \leq\left\|v_{\varepsilon}\right\|^{4}-\mu \int_{\Omega} v_{\varepsilon}^{4} d x \\
& \leq S^{2}-\frac{1}{K_{2}^{2}}\left(\pi^{2} \mu-8 \pi K_{1} \int_{0}^{R}\left|\sigma^{\prime}(r)\right|^{2} d r\right) \varepsilon+O\left(\varepsilon^{2}\right) .
\end{aligned}
$$


We take $\sigma(r)=\cos (\pi r / 2 R)$. Then $\int_{0}^{R}\left|\sigma^{\prime}(r)\right|^{2} d r=\pi^{2} / 8 R$. Hence taking $\varepsilon>0$ sufficiently small, we conclude that if $\mu>3 \pi^{3} / 4 R, K_{\mu}<S^{2}$. This gives the conclusion.

Finally we prove Theorem 4.2.

Proof of Theorem 4.2. First choose $3 \pi^{3} / 4 R<\mu<\mu_{1}$. Then $K_{\mu}<S^{2}$ by Lemma 4.5. Hence from Lemma 4.4, we have a nontrivial minimizer $u \in H_{0}^{1}(\Omega)$ of $K_{\mu}$. Thus there exists a Lagrange multiplier $\lambda \in \mathbb{R}$ such that $-\|u\|^{2} \Delta u-$ $\mu u^{3}=\lambda u^{5}$ in $\Omega$. We have further, $\lambda=K_{\mu}$. Since $\mu<\mu_{1}, K_{\mu}>0$ from the definition of $\mu_{1}>0$. Consequently, after an appropriate rescaling, we have the existence of a nontrivial and nonnegative solution of (K2). We can ensure the smoothness and positivity of the solution similarly to the argument in previous sections. This completes the proof.

\section{Appendix A. Nonexistence results}

In Appendix A, we prove the nonexistence results for $(\mathrm{K} 1)$, that is, we prove Theorem 1.5 and Theorem 1.6. To do that, we introduce a Kirchhoff type Pohozaev identity.

Lemma A.1. We assume $a, b \geq 0$ and $a+b>0$ and suppose $g$ satisfies (g1). Then if $u$ is a smooth solution of (K1), the following identity holds.

$$
\begin{gathered}
\frac{\left(a+b\|u\|^{2}\right)}{2}\|u\|^{2}-3 \mu \int_{\Omega} G(x, u) d x-\frac{1}{2} \int_{\Omega} u^{6} d x \\
+\frac{a+b\|u\|^{2}}{2} \int_{\partial \Omega}(x \cdot \nu)\left|\frac{\partial u}{\partial \nu}\right|^{2} d \sigma=0,
\end{gathered}
$$

where $\nu$ and $\partial / \partial \nu$ denote the outer normal vector and the outer normal derivative on $\partial \Omega$ respectively, and further $\sigma$ is the 2 dimensional surface measure on $\partial \Omega$.

Proof. Fix a solution $u$ of (K1). We define

$$
m\left(\|u\|^{2}\right):=\left(a+b\|u\|^{2}\right)^{-1}
$$

and

$$
f(x, u):=\mu g(x, u)+u^{5} .
$$

Then $u$ is a solution of

$$
\begin{cases}-\Delta u=m\left(\|u\|^{2}\right) f(x, u) & \text { in } \Omega \\ u=0 & \text { on } \partial \Omega\end{cases}
$$

The Pohozaev type identity for the solutions of the above equation is shown by a usual procedure. We get

$$
\frac{1}{2}\|u\|^{2}-3 m\left(\|u\|^{2}\right) \int_{\Omega} F(x, u) d x+\frac{1}{2} \int_{\partial \Omega}(x \cdot \nu)\left|\frac{\partial u}{\partial \nu}\right|^{2} d \sigma=0,
$$

where $F(x, u):=\int_{0}^{u} f(x, t) d t$. This concludes the proof.

Next we prove Theorem 1.5. 
Proof of Theorem 1.5. Let $a, b \geq 0, a+b>0,1<q<5$ and $g(x, t)=t^{q}$. In addition we suppose that $\mu \leq 0$ and $\Omega$ is strictly star-shaped. To conclude the proof, we assume that there exists a solution $u$ of (K1) to the contrary. Then we have

$$
\left(a+b\|u\|^{2}\right)\|u\|^{2}-\mu \int_{\Omega} u^{q+1} d x-\int_{\Omega} u^{6} d x=0 .
$$

Combining this relation and (A.1), we get

$$
\left(\frac{6}{q+1}-1\right) \mu \int_{\Omega} u^{q+1} d x=\left(a+b\|u\|^{2}\right) \int_{\partial \Omega}(x \cdot \nu)\left|\frac{\partial u}{\partial \nu}\right|^{2} d \sigma .
$$

If $\mu<0$, the right hand side of the above equality is strictly less than 0 . This is a contradiction since $x \cdot \nu>0$ on $\partial \Omega$. Now we assume $\mu=0$. Then we have from the above equality,

$$
\frac{\partial u}{\partial \nu}=0 \quad \text { on } \partial \Omega
$$

Therefore we have

$$
0=-\int_{\partial \Omega} \frac{\partial u}{\partial \nu} d \sigma=-\int_{\Omega} \Delta u d x=\frac{1}{a+b\|u\|^{2}} \int_{\Omega}\left(u^{q}+u^{5}\right) d x .
$$

But the right hand side of the above equality must be strictly positive. This is a contradiction. Hence there exists no solution for (K1) with $\mu \leq 0$. This is the desired conclusion.

Lastly we prove Theorem 1.6. We refer to the argument in [8].

Proof of Theorem 1.6. Assume $a>0$ and $b \geq 0$ and let $1<q \leq 3$ and $g(x, t)=t^{q}$. If $u$ is a solution of (K1), there holds

$$
\left(a+b\|u\|^{2}\right)\|u\|^{2}-\mu \int_{\Omega} u^{q+1} d x-\int_{\Omega} u^{6} d x=0 .
$$

By this relation and (A.1), we have

$$
\mu \int_{\Omega} u^{q+1} d x=C\left(a+b\|u\|^{2}\right) \int_{\partial \Omega}(x \cdot \nu)\left|\frac{\partial u}{\partial \nu}\right|^{2} d \sigma
$$

where $C>0$ is some constant depending only on $q$. Then by the assumption that $\Omega$ is strictly star shaped and the embedding $L^{2}(\partial \Omega) \hookrightarrow L^{1}(\partial \Omega)$, we have

$$
\begin{aligned}
\mu \int_{\Omega} u^{q+1} d x & \geq C\left(a+b\|u\|^{2}\right) \int_{\partial \Omega}\left|\frac{\partial u}{\partial \nu}\right|^{2} d \sigma \\
& \geq C\left(a+b\|u\|^{2}\right)\left(\int_{\partial \Omega} \frac{\partial u}{\partial \nu} d \sigma\right)^{2} \\
& =C\left(a+b\|u\|^{2}\right)\left(\int_{\Omega}|\Delta u| d x\right)^{2} \\
& \geq C\left(a+b\|u\|^{2}\right)[u]_{3, w}^{2}
\end{aligned}
$$

for some constant $C>0$ which depends only on $q$ and $\Omega$, where $[u]_{p, w}:=$ $\sup _{\lambda>0}\left[\lambda|\{u>\lambda\}|^{\frac{1}{p}}\right]$ denotes the $L^{p}$ weak norm of $u$ and $|A|$ denotes the 
Lebesgue measure of the set $A \subset \mathbb{R}^{3}$. Further here, for the third inequality of the above inequality we use the Green representation formula, the facts that $|x|^{-1}$ belongs to weak $L^{3}$ space and the Young's inequality for weak $L^{3}$ norm of convolution $|x|^{-1} *|\Delta u|$. On the other hand, since $\left(a+b\|u\|^{2}\right)|\Delta u| \geq u^{5}$, we have

$$
\mu \int_{\Omega} u^{q+1} d x \geq \frac{C}{a+b\|u\|^{2}}\left(\int_{\Omega} u^{5} d x\right)^{2} .
$$

Now define

$$
r:=\frac{6(q+1)}{q+3}
$$

Then clearly $q+1 \leq r$ and $3<r<5$. Put $0<\gamma<1$ so that

$$
\gamma:=\frac{3(5-r)}{2 r}
$$

Using $r, a, \gamma>0,(\mathrm{~A} .2)$ and (A.3) we get

$$
\begin{aligned}
\left(\int_{\Omega} u^{q+1} d x\right)^{\frac{1}{q+1}} \leq & C\left(\int_{\Omega} u^{r} d x\right)^{\frac{1}{r}} \\
\leq & C[u]_{3, w}^{\gamma}\left(\int_{\Omega} u^{5} d x\right)^{\frac{1-\gamma}{5}} \\
\leq & C\left\{\left(a+b\|u\|^{2}\right)^{-1} \mu \int_{\Omega} u^{q+1} d x\right\}^{\frac{\gamma}{2}} \\
& \times\left\{\left(a+b\|u\|^{2}\right) \mu \int_{\Omega} u^{q+1} d x\right\}^{\frac{1-\gamma}{10}},
\end{aligned}
$$

for some constant $C>0$ depending only on $q$ and $\Omega$. Since

$$
\frac{\gamma}{2}+\frac{1-\gamma}{10}=\frac{1}{q+1}
$$

we have

$$
\left(a+b\|u\|^{2}\right)^{\left(\frac{\gamma}{2}-\frac{1-\gamma}{10}\right)(q+1)} \leq C \mu .
$$

for some constant $C>0$ depending only on $q$ and $\Omega$. Since

$$
\frac{\gamma}{2}-\frac{1-\gamma}{10}>0
$$

we finally have

$$
\mu \geq \mu_{0}
$$

for some constant $\mu_{0}>0$ which depends only on $a, q$ and $\Omega$. This proves Theorem 1.6. 


\section{Acknowledgements}

The author is grateful for Institute of Mathematics, Academia Sinica where a part of his work was done during his visiting there. He appreciates to Professor F. Takahashi for his great supports and helpful suggestions for his research. He also thanks to M. Hashizume for his favorable discussion on a part of his result.

\section{References}

[1] Alves, C.O., Corrêa, F.J.S.A., Ma, T.F.: Positive solutions for a quasilinear elliptic equation of Kirchhoff type. Comput. Math. Appl. 49, 85-93 (2005)

[2] Alves, C.O., Corrêa, F.J.S.A., Figueiredo, G.M.: On a class of nonlocal elliptic problems with critical growth. Differ. Equ. Appl. 2, 409-417 (2010)

[3] Alves, C.O., Figueiredo, G.M.: Nonlinear perturbations of a periodic Kirchhoff equation in $\mathbb{R}^{N}$. Nonlinear Anal. 75, 2750-2759 (2012)

[4] Arosio, A.: Averaged evolution equations. The Kirchhoff string and its treatment in scales of Banach spaces. In: Functional Analytic Methods in Complex Analysis and Applications to Partial Differential Equations (Trieste, 1993), pp. 220-254. World Sci. Publ., River Edge (1995)

[5] Arosio, A., Panizzi, S.: On the well-posedness of Kirchhoff string. Trans. Am. Math. Soc. 348, 305-330 (1996)

[6] Azzollini, A., d'Avenia, P., Pomponio, A.: Multiple critical points for a class of nonlinear functionals. Ann. Mat. Pure Appl. 190, 507-523 (2011)

[7] Bernstein, S.: Sur une classe d'équations fonctionnelles aux dérivées partielles. Izk Akad. Nauk SSSR. Ser. Mat. 4, 17-26 (1940)

[8] Brezis, H.: Some variational problems with lack of compactness, Nonlinear functional analysis and its applications, part 1 (Berkeley, Calif., 1983). In: Proc. Simpos. Pure Math., vol. 45, pp. 165-201, Part 1. Amer. Math. Soc., Providence (1986)

[9] Brezis, H., Nirenberg, L.: Positive solutions of nonlinear elliptic equations involving critical Sobolev exponents. Commun. Pure Appl. Math. 36, 437-477 (1983)

[10] Carrier, G.F.: On the nonlinear vibration problem of the elastic string. Quart. Appl. Math. 3, 157-165 (1945)

[11] Carrier, G.F.: A note on the vibrating string. Quart. Appl. Math. 7, 97$101(1949)$

[12] Clark, D.C.: A variant of the Ljusternik-Schnirelmann theory. Indiana Univ. Math. J. 22, 65-74 (1972)

[13] D'Ancona, P., Spagnolo, S.: Global solvability for degenerate Kirchhoff equation with real analytic date. Invent. Math. 108, 247-262 (1992) 
[14] Figueiredo, G.M.: Existence of a positive solution for Kirchhoff problem type with critical growth via truncation argument. J. Math. Anal. Appl. 401, 706$713(2013)$

[15] Figueiredo, G.M., Santos, J.R. Jr.: Multiplicity of solutions for a Kirchhoff equation with subcritical or critical growth. Differ. Int. Equ. 25, 853-868 (2012)

[16] Gilbarg, D.; Trudinger, N.S.: Elliptic Partial Differential Equations of Second Order. Springer, Berlin (2001)

[17] Jeanjean, L., Le Coz, S.: An existence and stability result for standing waves of nonlinear Schrödinger equations. Adv. Differ. Equ. 11, 813-840 (2006)

[18] Kikuchi, H.: Existence and stability of standing waves for Schrödinger-PoissonSlater equation. Adv. Nonlinear Stud. 7, 403-437 (2007)

[19] Kirchhoff, G.: Vorlesungen über mathematische Physik: Mechanik, Teubner, Leipzig (1876)

[20] Li, Y., Li, F., Shi, J.: Existence of a positive solution to Kirchhoff type problems without compactness conditions. J. Differ. Equ. 253, 2285-2294 (2012)

[21] Liang, Z., Li, F, Shi, J.: Positive solutions to Kirchhoff type equations with nonlinearity having prescribed asymptotic behavior. Ann. Inst. H. Poincaré Anal. Non Linéaire (2013, in Press)

[22] Liang, S., Shi, S.: Soliton solutions to Kirchhoff type problems involving the critical growth in $\mathbb{R}^{N}$. Nonlinear Anal. 81, 31-41 (2013)

[23] Lions, J.L.: On some questions in boundary value problems of mathematical physics. In: de la Penha, G.M., Medeiros, L.A. (eds.) Contemporary Developments in Continuum Mechanics and PDE's. North-Holland, Amsterdam (1978)

[24] Lions, P.L.: The concentration-compactness principle in the calculus of variations The limit case. Part 1. Rev. Mat. Iberoamericana 1, 145-201 (1985)

[25] Ma, T.F., Muñoz Rivera, J.E.: Positive solutions for a nonlinear nonlocal elliptic transmission problem. Appl. Math. Lett. 16, 243-248 (2003)

[26] Narashima, R.: Nonlinear vibration of an elastic string. J. Sound Vib. 8, 134146 (1968)

[27] Oplinger, D.W.: Frequency response of a nonlinear stretched string. J. Acoust. Soc. Am. 32, 1529-1538 (1960)

[28] Perera, K., Zhang, Z.: Nontrivial solutions of Kirchhoff-type problems via the Yang index. J. Differ. Equ. 221, 246-255 (2006)

[29] Pohozaev, S.I.: Eigenfunctions of the equation $\Delta u+\lambda f(u)=0$. Soviet Math. Doklady 6, 1408-1411 (1965)

[30] Pohozaev, S.I.: On a class of quasilinear hyperbolic equations. Math. Ussr Sbornik 25, 145-158 (1975) 
[31] Schlesinger, K.: Saitenschwingungen mit endlicher amplitude. Z. Techn. Phys. 12, 33-39 (1931)

[32] Struwe, M.: Variational Methods: Applications to Nonlinear Partial Differential Equations and Hamiltonian Systems. 4th ed. Springer, Berlin (2008)

[33] Sun, Y., Liu, X.: Existence of positive solutions for Kirchhoff type problems with critical exponent. J. Partial Differ. Equ. 25, 187-198 (2012)

[34] Willem, M.: Minimax Theorems. Birkhäuser, Basel (1996)

[35] Wang, J., Tian, L., Xu, J., Zhang, F.: Multiplicity and concentration of positive solutions for a Kirchhoff type problem with critical growth. J. Differ. Equ. 253, 2314-2351 (2012)

Daisuke Naimen

Department of Mathematics

Graduate School of Science

Osaka City University

3-3-138 Sugimoto Sumiyoshi-ku

Osaka-shi

Osaka 558-8585

Japan

e-mail: d12sax0J51@ex.media.osaka-cu.ac.jp

Received: 14 August 2013.

Revised: 3 March 2014.

Accepted: 10 March 2014. 